\title{
The high-density regime of kinetic-dominated loop quantum cosmology
}

\author{
M. Bojowald* and W. Nelson ${ }^{\dagger}$ \\ Institute for Gravitation and the Cosmos, Penn State University, State College, PA 16801, U.S.A. \\ D. Mulryne \\ Department of Physics, Imperial College London, \\ South Kensington campus, London, SWr 2AZ. U.K. \\ R. Tavakol ${ }^{\S}$ \\ School of Mathematical Sciences, Queen Mary University of London, London E1 4NS, U.K.
}

\begin{abstract}
We study the dynamics of states perturbatively expanded about a harmonic system of loop quantum cosmology, exhibiting a bounce. In particular, the evolution equations for the first and second order moments of the system are analyzed. These moments back-react on the trajectories of the expectation values of the state and hence alter the energy density at the bounce. This analysis is performed for isotropic loop quantum cosmology coupled to a scalar field with a small but non-zero constant potential, hence in a regime in which the kinetic energy of matter dominates. Analytic restrictions on the existence of dynamical coherent states and the meaning of semi-classicality within these systems are discussed. A numerical investigation of the trajectories of states that remain semi-classical across the bounce demonstrates that, at least for such states, the bounce persists and that its properties are similar to the standard case, in which the moments of the states are entirely neglected. However the bounce density does change, implying that a quantum bounce may not be guaranteed to happen when the potential is no longer negligible.
\end{abstract}

\section{INTRODUCTION}

Loop quantum cosmology [1] imports mathematical constructions and results obtained in the general setting of quantum geometry realized in loop quantum gravity [2 -4] into quantum cosmology. As a result, the quantum representation is unitarily inequivalent to the one used in Wheeler-DeWitt quantizations of cosmological models, a formal difference entailing also dynamical changes in the behavior of models for the very early universe. Loop quantum cosmology thus allows one to explore the physical implications of quantum geometry and its unconventional space-time structures in a tractable setting.

One of the main changes implied by loop quantum gravity is the use of holonomies [5], objects of the form $\exp (i \mathrm{curv})$ replacing the usual curvature expressions "curv" (extrinsic and intrinsic curvature, or space-time curvature components) in the classical equations. The form of the curvature functional "curv" appearing in holonomies depends on the specific quantization of the Hamiltonian constraint used [6] as well as on details of the reduction to a symmetric context within quantum gravity [7]; however, within isotropic cosmological models it is a function only of the scale factor and its time derivative. Moreover, neither is the full theory of loop quantum gravity uniquely and unambiguously formulated yet, nor is the reduction from general physical states to isotropic ones fully understood at a quantitative level. Despite such ambiguities, several general conclusions can be inferred from mathematical properties of the curvature-holonomy replacement, most importantly related to the boundedness of holonomy expressions in contrast to the curvature components. For instance, the Friedmann equation relates extrinsic curvature $a \dot{a}$ of spatial slices in a dynamical isotropic universe to the energy density of matter. If extrinsic curvature in the equation is replaced by a bounded function of a certain form, boundedness of the energy density automatically follows in spite of its classical divergence at the big bang singularity.

Replacing curvature expressions by bounded functions is not simply done by hand but is motivated by quantum theory. Quantization also means that one is dealing with states rather than classical geometries subject to the usual evolution equations. Fundamentally, the use of holonomies primarily implies that the dynamics of quantum states of universe models is governed not by the differential Wheeler-DeWitt equation [8, 9], but by a difference equation [10, 11]. These discrete dynamics turn out to be non-singular [12, 13]: wave functions extend uniquely across the classically singular big bang. But while the use of holonomies and the discreteness of the difference equation

\footnotetext{
* bojowald@gravity.psu.edu

$\dagger$ nelson@gravity.psu.edu

‡ d.mulryne@imperial.ac.uk

$\S$ r.tavakol@qmul.ac.uk
} 
implied by it are important for this result, detailed properties of the transition depend on various kinds of quantum effects that the use of the wave function introduces. In particular, in addition to quantum geometry effects such as discreteness, there are in general quantum back-reaction effects that characterize deviations of the quantum evolution of expectation values from the classical evolution caused by a changing wave function. Simple intuitive boundedness results on energy densities can be established only if quantum geometry effects can be shown not to be overpowered by potentially adverse quantum back-reaction effects. In loop quantum cosmology, this competition of different quantum effects has not been explored sufficiently in generic regimes. (Examples in Wheeler-DeWitt quantum cosmology have shown the sensitivity of some quantum effects related to singularity avoidance to state properties [14 16].)

Typically attention has been focused on harmonic models, in which the quantum back-reaction is entirely absent. This has the advantage of being analytically tractable and has been shown to generically lead to bouncing solutions. The robustness of these results for models that deviate from these special cases has only begun to be explored and in this paper we further develop a systematic perturbation theory approach to this question [17, 18]. Key results reported here include the interplay of reality conditions (physical normalization of states) and uncertainty relations, which in non-harmonic cases turns out to be important for consistent evolution, numerical or otherwise. By numerical studies, we demonstrate the sensitivity of bounce properties such as the density to quantum effects. Also the high sensitivity of evolution through the bounce on initial values, observed for harmonic models based on an analysis of dynamical coherent states [19, 20], is qualitatively confirmed in our more general setting. Although the leading-order perturbations used here do not allow quantum dynamical corrections large enough to (potentially) remove the bounce induced by the holonomy modification, the sensitivity we find appears sufficiently strong to warrant caution about extrapolations of the kinetic-dominated behavior to models with significant matter potentials.

\section{LOOP QUANTUM COSMOLOGY}

According to the holonomy replacement scheme, one of the basic objects in loop quantum cosmology is the holonomy $\exp (i$ curv $)$ with an expression "curv" linear in curvature components. For an isotropic cosmological model, this means curv $=g(a) \dot{a}$ in terms of the scale factor $a$, with an a-priori arbitrary function $g(a)$. Since holonomy operators in loop quantum gravity are related to the discrete quantum geometry, the specific form of $g(a)$ depends on how those structures are realized and how they reduce to the case of isotropic models, which is referred to as lattice refinement [21, 22]. At present, $g(a)$ does not appear to be fixed uniquely by reduction schemes from the full dynamics, and so we will parametrize it as a power law, $g(a)=g_{0} a^{2 x}$ with a dimensionless number $x$ and a constant $g_{0}$ whose dimensions are such that $g(a) \dot{a}$ in the holonomy exponential is dimensionless. The dimension of $g_{0}$ thus depends on the value of $x$. (Several types of phenomenological analysis, mainly in the context of early-universe cosmology, have put restrictions on the ranges of parameters [23 27]).

\section{A. Canonical variables and a harmonic system}

Holonomies are used in loop quantum gravity not at the level of equations of motion but at the level of constraints which determine the dynamics of phase-space points. In the present context (spatially flat isotropic models), we have canonical variables $\tilde{c}=\gamma \dot{a}$ and its conjugate $|\tilde{p}|=a^{2}:\{\tilde{c}, \tilde{p}\}=8 \pi \gamma G / 3 V_{0}$ with the Barbero-Immirzi parameter $\gamma$ [28, 29], the gravitational constant $G$ and the coordinate size $V_{0}$ of a finite region chosen to integrate the full symplectic structure specialized to homogeneous fields. (Geometrically, the momentum $\tilde{p}$ actually refers to a densitized triad and thus can take both signs, depending on the orientation of the triad. In this article, we will assume $\tilde{p}$ to be positive without loss of generality.) The parameter $V_{0}$ is made implicit by absorbing it into the phase-space variables, $c:=V_{0}^{1 / 3} \tilde{c}$ and $p:=V_{0}^{2 / 3} \tilde{p}$, but one still must ensure that final results depend only on ratios of $c$ and $p$ independent of rescaling by $V_{0}$. See Appendix B for an explicit discussion of this so-called fiducial volume.

With these canonical variables, the holonomies we will use $\operatorname{are} \exp (i f(p) c)$, where $f(p)=f_{0} p^{x}$ is obtained simply by rearranging the previous parameters in $g(a)$ : for $f_{0} p^{x} c=f(p) c=g(a) \dot{a}=g_{0} a^{2 x} \dot{a}$, we identify $f_{0}=\gamma^{-1} V_{0}^{-(2 x+1) / 3} g_{0}$. With this $V_{0}$-dependence of $f_{0}$ we ensure that the product $f(p) c$ appearing in holonomies is independent of $V_{0}$, in addition to being dimensionless. It will be useful to apply a canonical transformation such that the combination $f(p) c$ appears as a canonical variable, which must then be conjugate to

$$
V:=\frac{3 p}{8 \pi \gamma G(1-x) f(p)}=\frac{3 a^{2(1-x)} V_{0}^{2(1-x) / 3}}{8 \pi \gamma G(1-x) f_{0}} \quad \text { such that } \quad\left\{f_{0} p^{x} c, V\right\}=1 .
$$

Since $f(p)$ scales by a factor of $V^{-1 / 3}, V \propto p / f(p)$ scales by a factor of $V_{0}$ if $V_{0}$ is changed. The volume of our fiducial 
cell, vol $=a^{3} V_{0}$, is then obtained as:

$$
\mathrm{vol}=\left(\frac{8 \pi \gamma G(1-x) f_{0}}{3} V\right)^{\frac{3}{2(1-x)}}
$$

In addition to $f(p) c$ used in holonomies, another combination independent of $V_{0}$ is $f(p) \sqrt{p}=f_{0} p^{(1+2 x) / 2}$. The physical meaning of this quantity is the lattice spacing $L$ in an underlying discrete state that gives rise to the dynamics captured in the isotropic model. In terms of this parameter, we can write holonomies as $\exp (i \gamma L \mathcal{H})$ with the Hubble parameter $\mathcal{H}$. The lattice spacing can also be obtained as the ratio vol $/ V=8 \pi \gamma G(1-x) L / 3$, written as

$$
\frac{\mathrm{vol}}{V}=\frac{8 \pi \gamma G(1-x)}{3} \tilde{f}_{0} \tilde{p}^{\frac{1+2 x}{2}}
$$

in terms of the $V_{0}$-independent $\tilde{f}_{0}:=f_{0} V_{0}^{(1+2 x) / 3}$. This equation shows that $L$ is independent of the scale factor only for $x=-\frac{1}{2}$.

Via holonomies, the combination $f(p) c$ appears in dynamical equations, chiefly the Hamiltonian constraint which follows from multiplying the Friedmann equation by $a^{3}$. In canonical variables, this provides

$$
-\frac{1}{\gamma^{2}} c^{2} \sqrt{|p|}+\frac{8 \pi G}{3} H_{\text {matter }}=0
$$

with the matter Hamiltonian $H_{\text {matter }}$ typically depending on $p$ as well as on matter degrees of freedom. After the holonomy replacement, this equation reads

$$
-\frac{\sin ^{2}(f(p) c) \sqrt{|p|}}{\gamma^{2} f^{2}(p)}+\frac{8 \pi G}{3} H_{\text {matter }}=0
$$

picking the sine as a real combination to be specific. (The precise form of this function replacing $c^{2}$, restricted only to be bounded as a linear combination of $\exp ( \pm i f(p) c)$ and to approach $c^{2}$ for small $f(p) c$ in a low-curvature regime, can be motivated from full quantizations of curvature [11, 30, 31] but is not determined uniquely. Further quantization ambiguities arise at this stage in addition to those in the form of $f(p)$.)

When quantized, Eq. (4), provides the difference equation of loop quantum cosmology. For general matter fields the resulting behavior can be hard to analyze, but in some special cases the dynamics becomes exactly solvable. This is the case in particular for a free, massless scalar $\phi$ with Hamiltonian $H_{\text {matter }}=\frac{1}{2} p^{-3 / 2} p_{\phi}^{2}$ in terms of the momentum $p_{\phi}$ conjugate to $\phi$. Quantizing the system, subject to a particular factor ordering then results in harmonic dynamics [32, 33], which leads to an evolution that is not subject to quantum back-reaction; in the sense that while states may spread and deform as they evolve, the changing shape does not affect the motion of expectation values. Hence quantum geometry corrections are dominant, resulting in the quantum bounce and the boundedness results.

Harmonicity is realized thanks to a linear algebra of a complete set of operators including the Hamiltonian in deparameterized form. We thus first use the free scalar as an internal time variable, formulating evolution not with respect to proper time, so far appearing in derivatives denoted by the dot, but with respect to $\phi$. This requires the monotonicity of $\phi(t)$, which is globally guaranteed in some special cases including a constant (or vanishing) scalar potential, which are the cases considered below. Evolution for the relational quantities $p(\phi)$ and $c(\phi)$ is then generated by the Hamiltonian $p_{\phi}(c, p)$ obtained as a phase-space function by solving (4) for $p_{\phi}$ in $H_{\text {matter }}$. We obtain $p_{\phi}= \pm \sqrt{16 \pi G / 3}(1-x)|\operatorname{Im} J|$ with

$$
J:=V \exp \left(i f_{0} p^{x} c\right) .
$$

The Hamiltonian is linear in $J$, and $J$ forms a linear algebra with $V$ by taking Poisson brackets:

$$
\{V, J\}=-i J \quad, \quad\{V, \bar{J}\}=i \bar{J} \quad, \quad\{J, \bar{J}\}=2 i V .
$$

Classical equations of motion are linear and easy to solve, but even the quantum dynamics based on the Hamiltonian $\hat{H}:=|\operatorname{Im} \hat{J}|$ (dropping the constant $\sqrt{16 \pi G / 3}(1-x)$ for simplicity, i.e. rescaling our "time" $\phi$ ) and the commutator algebra

$$
[\hat{V}, \hat{J}]=\hbar \hat{J} \quad, \quad\left[\hat{V}, \hat{J}^{\dagger}\right]=-\hbar \hat{J}^{\dagger} \quad, \quad\left[\hat{J}, \hat{J}^{\dagger}\right]=-2 \hbar(\hat{V}+\hbar / 2)
$$


simplifies strongly. ${ }^{1}$ For instance, equations of motion $\mathrm{d}\langle\hat{V}\rangle / \mathrm{d} \phi=\langle[\hat{V}, \hat{H}]\rangle / i \hbar$ and $\mathrm{d}\langle\hat{J}\rangle / \mathrm{d} \phi=\langle[\hat{J}, \hat{H}]\rangle / i \hbar$ for expectation values are fully determined by the expectation values and remain linear. ${ }^{2}$ This feature, shared with the harmonic oscillator in quantum mechanics, implies the absence of quantum back-reaction i.e. expectation values evolve irrespective of other moments of a state.

\section{B. Small potential}

Harmonic models are very simple but also extremely special. For robust conclusions, one must at least see how slight deviations from solubility might change the conclusions derived in this simple case. In the present context, the main ingredient for realistic modeling is the inclusion of a non-trivial matter potential. A number of key features change when the scalar potential no longer vanishes. First, $\phi$ can at best be taken as a local internal time since it may develop turning points in a varying potential. Secondly, the Hamiltonian is no longer in a linear algebra with basic operators, equations of motion for expectation values no longer form a closed set, and quantum back-reaction ensues. Dynamics can no longer be formulated in terms of expectation values alone; we must include at least a certain number of moments of the evolving state, which, following [17], we define as

$$
\underbrace{V \cdots V}_{\text {a }} \underbrace{J \cdots J}_{b}=\left\langle(\hat{V}-\langle\hat{V}\rangle)^{a}(\hat{J}-\langle\hat{J}\rangle)^{b}\right\rangle_{\text {Weyl }}
$$

for all positive integers $a$ and $b$ such that $a+b \geq 2$, indicating the totally symmetric ordering of operators by the subscript "Weyl". In general, all these moments are coupled dynamically to the expectation values, providing a complicated infinite-dimensional dynamical system. Approximately, it becomes tractable when an initial semiclassical state is involved, whose higher moments are suppressed by powers of $\hbar^{1 / 2}$ compared to lower ones. We will make use of this approximation in our analysis.

In the presence of a non-vanishing potential $\tilde{W}(\phi)$, the expression for $p_{\phi}$ on the constraint surface satisfying

$$
\frac{4 \pi G}{3} \frac{p_{\phi}^{2}}{\operatorname{vol}}+\operatorname{vol} \tilde{W}=\frac{\operatorname{vol}^{1 / 3}\left(\operatorname{Im} e^{i f(p) c}\right)^{2}}{\gamma^{2} f(p)^{2}}
$$

is

$$
p_{\phi}= \pm \sqrt{\frac{16 \pi G}{3}}(1-x) \sqrt{(\operatorname{Im} J)^{2}-\frac{3}{8 \pi G(1-x)^{2}}\left(\frac{8 \pi \gamma G(1-x)}{3} f_{0} V\right)^{3 /(1-x)} \tilde{W}} .
$$

If we define

$$
W(\phi):=\frac{3}{8 \pi G(1-x)^{2}}\left(\frac{8 \pi \gamma G(1-x)}{3} f_{0}\right)^{3 /(1-x)} \tilde{W}
$$

and absorb the same factor in $p_{\phi}$ as in the free case, we see that the momentum is proportional to

$$
H(V, J):=\sqrt{(\operatorname{Im} J)^{2}-V^{3 /(1-x)} W(\phi)},
$$

constituting a non-linear and typically "time"-dependent Hamiltonian. The rescaled potential $W$ depends on $V_{0}$ (via $\left.f_{0}\right)$ in such a way that $V^{3 /(1-x)} W$ behaves like $V_{0}^{2}$.

We will assume the potential to be always small compared to the kinetic energy of the scalar, putting us in the kinetic-dominated regime of loop quantum cosmology, close to the harmonic model. Sine the harmonic model has a

${ }^{1}$ Changing $V_{0}$ is subtle at the quantum level. Classically, the Poisson bracket is not preserved, implying that the transformation is not canonical. The non-canonical classical transformation then is not implemented unitarily. One can mimic the change of the Poisson bracket by a rescaling of $\hbar$, which does not amount to a physical change of its value but rather of the representation. Observables even at the quantum level do not depend on $V_{0}$ when these properties are taken into account.

${ }^{2}$ With the absolute value in $\hat{H}=|\operatorname{Im} \hat{J}|$, the Hamiltonian is not strictly linear. However, the Hamiltonian is preserved in the case of a $\phi$-independent potential, and so one only has to ensure that an initial state is supported solely on the positive (or negative) part of the spectrum of $\operatorname{Im} \hat{J}$ and the absolute value can be dropped for all the evolution. For a state required only to be semiclassical at one time, the spectral condition can easily be arranged. Alternatively, effective-constraint techniques [34 36] exist which allow one to deal directly with the constraint, avoiding deparameterization and taking square roots. 
large class of states that remain semiclassical (or even coherent) for long times even across the bounce, the kineticdominated semiclassical regime of anharmonic models can be expected to allow long evolution as well, an expectation to be confirmed in this article. Larger potentials, for which states and bounce properties can change more drastically, will require significantly more care.

For a small potential, we can expand the square root, but terms containing the potential remain non-linear in $V$ and $J$. Adding the potential to the system thus provides an anharmonicity, and the exact solubility properties of the free model disappear. Not only the classical but also the quantum dynamics become much more complicated and in general can be treated only by using approximations. Even if it is possible to define the quantum dynamics in deparameterized form, such as with a constant $W$ as used below, we have to face the difficulty of defining a complicated square-root operator for $p_{\phi}$.

At this stage, effective equations offer valuable tools to approximate the quantum evolution of general semiclassical states, sidestepping many difficulties. For a semiclassical state, we can expand the quantum Hamiltonian

$$
\begin{aligned}
H_{Q}\left(V, J, G^{a, b}\right) & :=\langle H(\hat{V}, \hat{J})\rangle=\langle H(\langle\hat{V}\rangle+(\hat{V}-\langle\hat{V}\rangle),\langle\hat{J}\rangle+(\hat{J}-\langle\hat{J}\rangle))\rangle \\
& =H(\langle\hat{V}\rangle,\langle\hat{J}\rangle)+\sum_{a, b: a+b \geq 2} \frac{1}{a ! b !} \frac{\partial^{a+b} H(\langle\hat{V}\rangle,\langle\hat{J}\rangle)}{\partial\langle\hat{V}\rangle^{a} \partial\langle\hat{J}\rangle^{b}} G^{a, b}
\end{aligned}
$$

as a Hamiltonian function on the quantum phase space of states parameterized by the expectation values $V:=\langle\hat{V}\rangle$ and $J:=\langle\hat{J}\rangle$ together with the moments $G^{a, b}$. All the moments, as well as the expectation values, are subject to Poisson bracket relationships that are uniquely defined by $\{\langle\hat{A}\rangle,\langle\hat{B}\rangle\}=\langle[\hat{A}, \hat{B}]\rangle / i \hbar$; thus, the quantum Hamiltonian generates Hamiltonian equations of motion $\mathrm{d} F / \mathrm{d} \phi\left(V, J, G^{a, b}\right)=\left\{F, H_{Q}\right\}$ as usual. Also in this expansion the quantum Hamiltonian is clearly non-linear for a non-vanishing potential. Coupling terms between expectation values and moments imply quantum back-reaction. For semiclassical states, high moments are sub-dominant and the infinite sum can be truncated to finitely many terms, starting with quantum corrections due to fluctuations and correlations.

We note that our condition for semiclassical states is rather weak, and so the allowed class is very general, much more general than usually considered in evolutions of specific states. We only require that a moment of order $a+b+1$ is suppressed by an additional power of $\hbar^{1 / 2}$ compared to moments of order $a+b$. (For dimensional reasons, the behavior must then be $G^{a, b} \sim(V \hbar)^{(a+b) / 2}$ in our variables, $V$ and $J$ having the same dimension as $\hbar$.) This behavior is realized for Gaussian states (which fix all moments in terms of just one or two real parameters), but certainly not only in that case. States are allowed to spread out, deform their shape and deviate from Gaussians. As long as the hierarchy of moments is satisfied, the approximate quantum evolution remains reliable. In quantum cosmology, the evolution equations we use can hold true for sufficiently long times to answer long-term questions, and their validity can be tested self-consistently.

In the specific case at hand, the quantum Hamiltonian is

$$
\begin{aligned}
H_{Q}= & \frac{J-\bar{J}}{2 i}-i \frac{V^{3 /(1-x)}}{J-\bar{J}} W(\phi)-\frac{3}{2} i \frac{2+x}{(1-x)^{2}} \frac{V^{(1+2 x) /(1-x)}}{J-\bar{J}} G^{V V} W(\phi) \\
& +\frac{3 i}{1-x} \frac{V^{(2+x) /(1-x)}}{(J-\bar{J})^{2}}\left(G^{V J}-G^{V \bar{J}}\right) W(\phi) \\
& -i \frac{V^{3 /(1-x)}}{(J-\bar{J})^{3}}\left(G^{J J}-2 G^{J \bar{J}}+G^{\bar{J} \bar{J}}\right) W(\phi)
\end{aligned}
$$

to second order in the moments, combined with an expansion up to linear terms in the potential. Note that the energy of a system of physical volume, vol, is related to $H_{Q}$ as

$$
\text { Energy }=\frac{1}{2} \frac{p_{\phi}^{2}}{\operatorname{vol}}+\operatorname{vol} \tilde{W}=\frac{8 \pi G}{3}(1-x)^{2} H_{Q}^{2} / \operatorname{vol}+\operatorname{vol} \tilde{W},
$$

so $H_{Q} /$ vol is proportional to the square-root of the kinetic energy density.

In the following, $V$ and $J$ refer to expectation values of the basic operators in a state. Their products in interaction terms of the Hamiltonian thus are not subject to factor ordering ambiguities as they would if $H_{Q}$ were an operator. Also in the underlying Hamiltonian operator there are no ordering ambiguities since the expansion comes from a Hamiltonian quantizing (10), which lacks products of non-commuting operators. Although we have not written down an operator quantizing the square root in (10) explicitly, it is clear that the semiclassical expansion is unaffected by ordering choices. This statement holds provided that we choose $\hat{J}$ as one of our basic operators together with $\hat{V}$, as is done for the free harmonic model to make that system's Hamiltonian linear. Choosing $\hat{J}$ as the basic variable as (opposed to, say, $f(p) c)$ is consistent with our use of moments of $V$ and $J$. 
We note that many quantum effects can be summarized as an effective Friedmann equation [37, 38]

$$
\left(\frac{\dot{a}}{a}\right)^{2}=\frac{8 \pi G}{3}\left(\rho\left(1-\frac{\rho_{Q}}{\rho_{\text {crit }}}\right) \pm \frac{1}{2} \sqrt{1-\frac{\rho_{Q}}{\rho_{\text {crit }}}} \eta(\rho-P)+\frac{(\rho-P)^{2}}{\rho+P} \eta^{2}\right)
$$

with the critical energy density $\rho_{\text {crit }}=3 / 8 \pi G \gamma^{2} L^{2}$ and quantum corrections from correlations $\eta$ as well as fluctuations entering $\rho_{Q}$ (and $P$ is pressure). However, quantum correlations and fluctuations are determined by the moments of a state, and those moments are dynamical. An effective Friedmann equation thus provides a consistent set of differential equations only if it is accompanied by evolution equations for the moments. In this article, we are dealing from the outset with all equations for expectation values and moments to a certain order, bringing all quantum degrees of freedom under control.

\section{Non-dynamical conditions}

In addition to the dynamics of evolving moments, further conditions arise. First, to realize solubility of the potentialfree system we have chosen to work with a complex variable $J$, which classically must satisfy $|J|^{2}=V^{2}$ for the original phase-space variables $c$ and $p$ to be real. Reality conditions must also be imposed at the quantum level so that real phase-space variables are promoted to self-adjoint operators, ensuring that one is using the correct physical inner product to normalize states and compute expectation values and moments. After quantization, the reality condition still holds but is quantum corrected; we have

$$
|J|^{2}-\left(V+\frac{1}{2} \hbar\right)^{2}=G^{V V}-G^{J \bar{J}}+\frac{1}{4} \hbar^{2}
$$

from taking an expectation value of the quantum reality condition

$$
\hat{J} \hat{J}^{\dagger}=\hat{V}^{2}
$$

and expressing this in terms of moments and expectation values. The reality condition thus relates second-order moments to expectation values, but unlike the quantum Hamiltonian it is not truncated: it is an exact relationship for a finite number of our quantum variables. Note that in this expression one can neglect the $\hbar^{2}$ terms, because we work in the regime $V \gg \hbar$ and the second order moments are order $V \hbar$ and hence dominate the right hand side. However for clarity we will keep these terms and take the appropriate limits only at the end of calculations.

In addition to (16), which relates expectation values in a semiclassical state with second-order moments of order $\hbar$, reality conditions exist for the moments themselves. By taking the expectation values of Eq. (17), one arrives at Eq. (16), however one can find further independent conditions, implied by taking an expectation value for instance of $\hat{V} \hat{J} \hat{J}^{\dagger}=\hat{V}^{3}$. These new conditions relate third-order moments

$$
\begin{aligned}
G^{V J \bar{J}} & \equiv\left\langle(\hat{V}-\langle\hat{V}\rangle)(\hat{J}-\langle\hat{J}\rangle)\left(\hat{J}^{\dagger}-\left\langle\hat{J}^{\dagger}\right\rangle\right)\right\rangle_{\mathrm{Weyl}} \\
G^{V V V} & \equiv\left\langle(\hat{V}-\langle\hat{V}\rangle)^{3}\right\rangle
\end{aligned}
$$

to second-order ones and expectation values. Our truncation considers the evolution equations only up to secondorder moments, disregarding third-order or higher ones, nevertheless, reality conditions obtained at third order restrict allowed choices for second-order moments, and thus will be important irrespective of the precise values of third-order moments. For instance, we obtain

$$
G^{V J \bar{J}}-G^{V V V}=\left(2 V+\frac{\hbar}{2}\right) G^{V V}-2\left(\operatorname{Re} G^{V J} \operatorname{Re} J+\operatorname{Im} G^{V J} \operatorname{Im} J\right)-\frac{\hbar}{2}\left(V^{2}-\frac{\hbar}{3} V-\frac{\hbar^{2}}{3}\right),
$$

an equation which contains terms of different orders in $\hbar$. Products such as $V G^{V V}$ are of order $V^{2} \hbar$ in a semiclassical state, while third-order moments behave like $(V \hbar)^{3 / 2}$ and are thus smaller. The leading order in $\hbar$ then implies a reality condition

$$
\begin{aligned}
V G^{V V} & =\operatorname{Re}\left(\bar{J} G^{V J}\right)+\mathcal{O}\left(\hbar^{3 / 2}\right) \\
& =\operatorname{Re} J \operatorname{Re} G^{V J}+\operatorname{Im} J \operatorname{Im} G^{V J}+\mathcal{O}\left((V \hbar)^{3 / 2}\right)
\end{aligned}
$$


for second-order moments. By analogous calculations, we obtain

$$
V G^{V J}=\frac{1}{2}\left(J G^{J \bar{J}}+\bar{J} G^{J J}\right)+\mathcal{O}\left((V \hbar)^{3 / 2}\right)
$$

from the leading order of an expectation value of $\hat{J}^{2} \hat{J}^{\dagger}=\hat{J} \hat{V}^{2}$. Eq. (22) is a complex equation, giving two real conditions

$$
\begin{aligned}
V \operatorname{Re} G^{V J} & =\frac{1}{2}\left(\operatorname{Re} J \operatorname{Re} G^{J J}+\operatorname{Im} J \operatorname{Im} G^{J J}+\operatorname{Re} J G^{J \bar{J}}\right)+\mathcal{O}\left((V \hbar)^{3 / 2}\right) \\
V \operatorname{Im} G^{V J} & =\frac{1}{2}\left(\operatorname{Re} J \operatorname{Im} G^{J J}-\operatorname{Im} J \operatorname{Re} G^{J J}+\operatorname{Im} J G^{J \bar{J}}\right)+\mathcal{O}\left((V \hbar)^{3 / 2}\right) .
\end{aligned}
$$

Similarly, low-order moments arise in expectation values of products with even more factors multiplying $\hat{J}^{\dagger}-\hat{V}^{2} .^{3}$ One could worry that arbitrarily high orders must be considered in order to find all reality conditions at second order. Fortunately, a combinatorial argument shows that this is not the case, and the reality conditions found here for second-order moments are complete. This conclusion can also be supported by a simple counting of degrees of freedom: We expect three independent second-order moments once all reality conditions are implemented: two fluctuations and one correlation parameter. Counting real and imaginary parts separately, we start with six moments of the $(V, J)$-variables $\left(G^{V V}\right.$ and $G^{J \bar{J}}$ are always real, in contrast to $G^{V J}$ and $\left.G^{J J}\right)$. With three reality conditions for the moments, a real one (21) and one complex one (22), the correct number results.

In addition to reality conditions, moments of a state must satisfy uncertainty relations. In our case of a noncanonical set of basic operators, there are three independent conditions for the second-order moments, which can be derived by standard means:

$$
\begin{aligned}
2 G^{V V}\left(\operatorname{Re} G^{J J}+G^{J \bar{J}}\right)-4\left(\operatorname{Re} G^{V J}\right)^{2} & \geq \hbar^{2}(\operatorname{Im} J)^{2}, \\
2 G^{V V}\left(-\operatorname{Re} G^{J J}+G^{J \bar{J}}\right)-4\left(\operatorname{Im} G^{V J}\right)^{2} & \geq \hbar^{2}(\operatorname{Re} J)^{2}, \\
\left(G^{J \bar{J}}\right)^{2}-\left(\operatorname{Re} G^{J J}\right)^{2}-\left(\operatorname{Im} G^{J J}\right)^{2} & \geq \hbar^{2}(V+\hbar / 2)^{2} .
\end{aligned}
$$

Note that for near-saturation these equations are consistent with the behavior $G^{a, b} \sim V \hbar$ of second-order moments with $a+b=2$.

\section{Evolution of the Moments}

Following [40] we obtain equations of motion for all the moments, in addition to quantum-corrected equations for the expectation values, by taking Poisson brackets with the quantum Hamiltonian. These equations, even when truncated to second order in the moments, are long, and for that reason given only in Appendix $\mathrm{A}$, but as a finite-dimensional dynamical system they are amenable to analytical as well as numerical investigations. The most important one of the equations is the evolution of $V$, since this will determine the conditions under which a bounce occurs; it is given by

$$
\begin{aligned}
\frac{\mathrm{d} V}{\mathrm{~d} \phi}= & -\frac{J+\bar{J}}{2}+\frac{J+\bar{J}}{(J-\bar{J})^{2}} V^{3 /(1-x)} W(\phi)-\frac{2 V^{3 /(1-x)}}{(J-\bar{J})^{3}}\left(G^{J J}-G^{\bar{J} \bar{J}}\right) W(\phi) \\
& -\frac{6}{1-x} \frac{J+\bar{J}}{(J-\bar{J})^{3}} V^{(2+x) /(1-x)}\left(G^{V J}-G^{V \bar{J}}\right) W(\phi) \\
& +\frac{3}{1-x} \frac{V^{(2+x) /(1-x)}}{(J-\bar{J})^{2}}\left(G^{V J}+G^{V \bar{J}}\right) W(\phi) \\
& +\frac{3}{2} \frac{2+x}{(1-x)^{2}} \frac{J+\bar{J}}{(J-\bar{J})^{2}} V^{(1+2 x) /(1-x)} G^{V V} W(\phi) \\
& +3 \frac{J+\bar{J}}{(J-\bar{J})^{4}} V^{3 /(1-x)}\left(G^{J J}+G^{\bar{J} \bar{J}}-2 G^{J \bar{J}}\right) W(\phi) .
\end{aligned}
$$

\footnotetext{
${ }^{3}$ Interpreting $J \bar{J}-V^{2}=0$ as a constraint on the classical phase space, this large set of expectation values is an example of effectiveconstraint methods developed in [34 [36]. The reality condition, although just one constraint, is of second class on the non-symplectic phase space spanned by $(V, J, \bar{J})$, using generalizations of Dirac's classification of constraints to general Poisson manifolds [39]. Thus, only conditions for the moments result but no gauge flow need be factored out.
} 
In total there are nine evolution equations for the real variables

$$
\left(V, \operatorname{Re} J, \operatorname{Im} J, G^{V V}, G^{J \bar{J}}, \operatorname{Re} G^{V J}, \operatorname{Im} G^{V J}, \operatorname{Re} G^{J J}, \operatorname{Im} G^{J J}\right)
$$

but not all the variables are independent owing to the non-dynamical conditions: the reality condition (16) combined with restrictions from the uncertainty relations. Moreover, (21) and (22), while not presenting sharp conditions when third-order moments are not specified, provide ranges allowed for the moments of an initial semiclassical state. Imposing the reality condition (16) leaves eight free variables. In addition, we will require that the uncertainty conditions are nearly saturated at least for an initial state. As a sharp condition, this can be used to fix the initial values of, e.g., $\operatorname{Re} G^{V J}, \operatorname{Im} G^{V J}$ and $G^{J \bar{J}}$, while the reality condition can be used to eliminate (for example) Im $J$, thus leaving five variables: $V, \operatorname{Re} J, G^{V V}, \operatorname{Re} G^{J J}$ and $\operatorname{Im} G^{J J}$. The relationship for the deparameterized Hamiltonian, $H_{Q} \propto p_{\phi}$, constitutes another equation which could be used to eliminate one further variable in favor of $p_{\phi}$. (But note that $p_{\phi}$ is a constant of motion only in the case of a constant potential; otherwise it is subject to its own, potentially complicated dynamics.) The remaining moments then constitute two fluctuation parameters, $G^{V V}$ and $\operatorname{Re} G^{J J}$, and one correlation parameter $\operatorname{Im} G^{J J}{ }^{4}$ For numerical evolution, we will choose initial values for these variables and ensure that this is compatible with (21) and (22).

Of particular interest is the evolution of the correlation parameter $\operatorname{Im} G^{J J}$ because its value plays a role in seeing whether the bounce of the free system generically remains if it is perturbed by the potential. Moreover, it contributes a term to the effective Friedmann equation (15) similar to a positive effective potential [37, 38]. Effective equations are not yet available in complete form for a non-trivial potential $W(\phi)$, in whose presence $\phi$ would not serve as global internal time. Additional corrections can be expected in that case, but they can be shown to be small for a sufficiently flat potential [4]. In our numerical discussions below (Sec. $\mathrm{V}$ ), we avoid this issue altogether by working with constant (but non-zero) potentials, sufficiently small to ensure kinetic domination as used in the expansions. Our goals are to provide a more systematic analysis of consequences of potential terms compared to what is available so far. In particular, we will analyze the effects of a non-zero potential on the appearance of bounces and the evolution of the moments.

\section{ANALYTIC RESTRICTIONS}

Typically one is concerned with a state that is semi-classical initially, which in this language means that the moments are small compared to the expectation value. It is useful then to define the fractional moments,

$$
\Delta G^{V V} \equiv \frac{G^{V V}}{V^{2}}, \quad \Delta G^{J \bar{J}} \equiv \frac{G^{J \bar{J}}}{|J|^{2}}, \quad \Delta G^{V J} \equiv \frac{G^{V J}}{V|J|}, \Delta G^{J J} \equiv \frac{G^{J J}}{|J|^{2}},
$$

and analogously for higher-order moments $\Delta G^{a, b}$. Fractional moments in a semiclassical state then behave as $\Delta G^{a, b} \sim$ $(\hbar / V)^{(a+b) / 2}$.

Note that the second-order moments $\Delta G^{V V}$ and $\Delta G^{J \bar{J}}$ (but only those) must be positive. In the free model, $V$ and $|J| \geq \operatorname{Im} J \propto p_{\phi}$ take macroscopic values at the bounce for large matter content in the kinetic-dominated regime, all fractional moments should thus be small if an evolving state remains semiclassical well into the bounce regime. The state remains semiclassical at all times in the free model, so that a sufficiently small anharmonicity brought about by the non-vanishing potential can be expected to allow semiclassical evolution into the bounce regime for a reasonably large class of states.

There are additional restrictions on specific combinations of the moments, imposed by uncertainty relations. In terms of the fractional moments, those relations, Eqs. (24)-(26), become,

$$
\begin{gathered}
2 \Delta G^{V V}\left(\operatorname{Re} \Delta G^{J J}+\Delta G^{J \bar{J}}\right)-4\left(\operatorname{Re} \Delta G^{V J}\right)^{2} \geq \hbar^{2} \frac{(\operatorname{Im} J)^{2}}{V^{2}|J|^{2}}, \\
2 \Delta G^{V V}\left(-\operatorname{Re} \Delta G^{J J}+\Delta G^{J \bar{J}}\right)-4\left(\operatorname{Im} \Delta G^{V J}\right)^{2} \geq \hbar^{2} \frac{(\operatorname{Re} J)^{2}}{V^{2}|J|^{2}},
\end{gathered}
$$

\footnotetext{
4 To classify second-order quantum variables as fluctuations or correlations, it is preferable to refer to the self-adjoint operators $\hat{J}_{+}:=$ $\frac{1}{2}\left(\hat{J}+\hat{J}^{\dagger}\right)$ and $\hat{J}_{-}:=\frac{1}{2 i}\left(\hat{J}-\hat{J}^{\dagger}\right)$ rather than $\hat{J}$ and $\hat{J}^{\dagger}$. Since this is a linear transformation, the order of moments is preserved. In

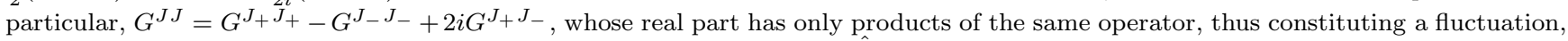
while the imaginary part contains the product of two different operators $\hat{J}_{ \pm}$.
} 


$$
\left(\Delta G^{J \bar{J}}\right)^{2}-\left(\operatorname{Re} \Delta G^{J J}\right)^{2}-\left(\operatorname{Im} \Delta G^{J J}\right)^{2} \geq \hbar^{2} \frac{(V+\hbar / 2)^{2}}{|J|^{4}}
$$

Using Eq. (16) and Eq. (32) we find an inequality for $V$,

$$
\begin{aligned}
V \geq & \frac{\hbar}{2 \mathcal{D}\left(1+\Delta G^{V V}\right)}\left[1+\Delta G^{J \bar{J}}-\mathcal{D}+\right. \\
& \left.\sqrt{\left(1+\Delta G^{J \bar{J}}-\mathcal{D}\right)^{2}+2 \Delta\left(1+\Delta G^{V V}\right)\left(1+\Delta G^{J \bar{J}}-\mathcal{D}\right)}\right]
\end{aligned}
$$

where

$$
\mathcal{D} \equiv \sqrt{\left(\Delta G^{J \bar{J}}\right)^{2}-\left(\operatorname{Re} \Delta G^{J J}\right)^{2}-\left(\operatorname{Im} \Delta G^{J J}\right)^{2}} .
$$

In addition, from Eq. (30) and Eq. (31), we find,

$$
V \geq \frac{1}{2 \sqrt{\Delta G^{V V} \Delta G^{J \bar{J}}-\left(\Delta \operatorname{Re} G^{V J}\right)^{2}-\left(\Delta \operatorname{Im} G^{V J}\right)^{2}}} .
$$

These two conditions must always be satisfied, and in particular they must be valid at the bounce, which is by definition the minimum value of the volume. Via Eq. (2) we find that the volume at the bounce is restricted. In particular, Eq. (35) implies that,

$$
\left.\left(\Delta G^{V V} \Delta G^{J \bar{J}}-\left(\operatorname{Re} \Delta G^{V J}\right)^{2}-\left(\operatorname{Im} \Delta G^{V J}\right)^{2}\right)\right|_{\text {Bounce }} \rightarrow 0
$$

can only occur for $V_{\text {bounce }} \rightarrow \infty$. Thus a semi-classical state (at the bounce) is not just one in which the fractional moments are small, since the uncertainty relations for such a state, would force the volume (of any spatial region at the time of the bounce) to be arbitrarily large. Second-order moments thus cannot be self-consistently ignored.

\section{A. Zero potential}

In this section we consider, in more detail, the case of a free scalar field, i.e. $W=0$, leaving the discussion of the $W \neq 0$ case to Section $\amalg$ TIIB The evolution of $V$ is given by Eq. (27) and in particular the bounce is given by $\frac{\mathrm{d} V}{\mathrm{~d} \phi}=0$ which gives,

$$
\left.\operatorname{Re} J\right|_{\text {Bounce }}=0
$$

Putting this into Eq. (16) we find,

$$
\left.\operatorname{Im} J\right|_{\text {Bounce }}=\left.\left[\frac{V^{2}\left(1+\Delta G^{V V}\right)+\hbar\left(V+\frac{\hbar}{2}\right)}{\left(1+\Delta G^{J \bar{J}}\right)}\right]^{1 / 2}\right|_{\text {Bounce }}=\left.H_{Q}\right|_{\text {Bounce }},
$$

where the second equality comes from Eq. (13). Thus we find the density,

$$
\left.\frac{H_{Q}}{\text { vol }}\right|_{\text {Bounce }}=\left.\left[\operatorname{vol}^{-1} \sqrt{\frac{V^{2}\left(1+\Delta G^{V V}\right)+\hbar\left(V+\frac{\hbar}{2}\right)}{\left(1+\Delta G^{J \bar{J}}\right)}}\right]\right|_{\text {Bounce }} .
$$

It is important to note that although this resembles an energy density, we have $H_{Q} \propto p_{\phi}$, and hence the energy density is the square of Eq. (39) (see the comments below Eq. (13)).

If we assume that at the bounce, $V$ is large ${ }^{5}$, i.e. that $V \gg \hbar$, then the density at the bounce is given by the

\footnotetext{
5 The variable $V$ depends on $V_{0}$ and can be made arbitrarily small (provided $x<1$ ) just by choosing a small $V_{0}$. But we have to be more careful at the quantum level due to the non-unitary implementation of changing $V_{0}$. For simplicity, we consider the limit of large $V$ as a substitute of the complete transformation, and ensure that physical values remain finite in the limit. (The limit of large $V_{0}$ has also been discussed in [41].)
} 
large- $V$ limit, which is just,

$$
\left.\frac{H_{Q}}{\text { vol }}\right|_{\text {Bounce, large } V}=\left.\left[\frac{3}{8 \pi G \gamma(1-x)} \tilde{f}_{0}^{-1} \tilde{p}^{-\frac{1+2 x}{2}} \sqrt{\frac{1+\Delta G^{V V}}{1+\Delta G^{J J}}}\right]\right|_{\text {Bounce, large } V},
$$

where the fact that $\left(1+\Delta G^{V V}\right) \geq 1$ and $\left(1+\Delta G^{J \bar{J}}\right) \geq 1$, ensures that the square root is real. Note that, as expected, this is independent of the fiducial volume $V_{0}$ (and $\tilde{f}_{0}^{-1} \tilde{p}^{-(1+2 x) / 2}=L^{-1}$ is constant in our limit of large $V$ ) and so is the bounce density

$$
\left.\rho\right|_{\text {Bounce, large } V}=\frac{3}{8 \pi G \gamma^{2} L^{2}} \frac{1+\Delta G^{V V}}{1+\Delta G^{J \bar{J}}}=\rho_{\text {crit }} \frac{1+\Delta G^{V V}}{1+\Delta G^{J \bar{J}}} .
$$

The $\tilde{p}$-independence of the bounce density for the specific case of $x=-1 / 2$ agrees with numerical results [42], as pointed out in [37] whose more general results are consistent also with the other cases of $x$. The specific dependence of the bounce density on the moments agrees with results obtained in the context of the effective Friedmann equation (15), where the energy density $\rho_{Q}=\rho+\epsilon_{0} \rho_{\text {crit }}+\cdots$, according to [37, 38], has the leading correction given by $\epsilon_{0}=\Delta G^{J \bar{J}}-\Delta G^{V V}$. There is a bounce when $\rho_{Q}=\rho_{\text {crit }}$, at which the density is $\rho=\rho_{\text {crit }}\left(1-\epsilon_{0}\right)$. For small fractional moments, this is exactly (41).

Notice that even for the $x=-\frac{1}{2}$ case, the energy density at the bounce still depends on the moments present at the bounce (even with $W=0$, i.e. zero potential). The presence of these moments can increase or decrease the energy density of the bounce compared to the case when they are neglected, however for semi-classical states, we can restrict the energy density to the range,

$$
\frac{1}{\sqrt{2}} \rho_{\text {crit }}<\left.\rho\right|_{\text {Bounce, large } V}<\sqrt{2} \rho_{\text {crit }},
$$

where $\rho_{\text {crit }}$ is the energy density of the bounce that would be calculated neglecting the effects of the moments and we consider a state to be semi-classical only if the factional moments are less than unity (admittedly, a rough estimate). The cases of equality can be removed by noting that Eq. (30) implies $\Delta G^{V V}>0$ and Eq. (32) gives $\Delta G^{J \bar{J}}>0$. This is a limit on all possible semi-classical states, for which the higher order moments can be neglected. If one wanted to consider a more strict definition of semi-classical states (i.e. states for which the fractional moments are below some specified value, less than one), the range would be further restricted.

\section{B. Non-zero potential}

For the more interesting case of $W \neq 0$, Eq. (27) and the bounce condition $\mathrm{d} V / \mathrm{d} \phi=0$ gives,

$$
\begin{aligned}
\operatorname{Re} J= & -\frac{W V^{\frac{3}{1-x}}}{\operatorname{Im} J}\left\{\frac{1}{2} \frac{\operatorname{Re} J}{\operatorname{Im} J}-\frac{3}{4} \frac{\operatorname{Re} J}{\operatorname{Im} J} \frac{|J|^{2}}{(\operatorname{Im} J)^{2}}\left[\operatorname{Re} \Delta G^{J J}-\Delta G^{J \bar{J}}\right]\right. \\
& -\frac{3}{1-x} \frac{\operatorname{Re} J}{\operatorname{Im} J} \frac{|J|}{(\operatorname{Im} J)^{2}} \operatorname{Im} \Delta G^{V J}+\frac{3}{4} \frac{2+x}{(1-x)^{2}} \frac{\operatorname{Re} J}{\operatorname{Im} J} \Delta G^{V V} \\
& \left.-\frac{1}{2} \frac{|J|^{2}}{(\operatorname{Im} J)^{2}} \operatorname{Im} \Delta G^{J J}+\frac{3}{2(1-x)} \frac{|J|}{\operatorname{Im} J} \operatorname{Re} \Delta G^{V J}\right\} .
\end{aligned}
$$

Changing to polar coordinates for the complex variable $J$ using,

$$
\sin \theta=\frac{\operatorname{Im} J}{|J|} \quad, \quad \cos \theta=\frac{\operatorname{Re} J}{|J|} \quad, \quad \tan \theta=\frac{\operatorname{Im} J}{\operatorname{Re} J},
$$

where $\theta=\arg (J)$, we can write Eq. (43) as

$$
\begin{aligned}
\cos \theta= & -\frac{W V^{\frac{3}{1-x}}}{|J|^{2} \sin \theta}\left\{\frac{\cos \theta}{2 \sin \theta}-\frac{3 \cos \theta}{4 \sin ^{3} \theta}\left[\operatorname{Re} \Delta G^{J J}-\Delta G_{J \bar{J}}\right]\right. \\
& -\frac{3 \cos \theta}{(1-x) \sin ^{2} \theta} \operatorname{Im} \Delta G^{V J}+\frac{3(2+x) \cos \theta}{4(1-x)^{2} \sin \theta} \Delta G^{V V} \\
& \left.-\frac{1}{2 \sin ^{2} \theta} \operatorname{Im} \Delta G^{J J}+\frac{3}{2(1-x) \sin \theta} \operatorname{Re} \Delta G^{V J}\right\} .
\end{aligned}
$$


Now we look for solutions that are 'close' to the $W=0$ case in the near-bounce regime, by expanding around $\theta=\pi / 2$ i.e. $\theta=\pi / 2+\delta \theta$, which gives $\sin \theta=1+\mathcal{O}\left(\delta \theta^{2}\right)$ and $\cos \theta=-\delta \theta+\mathcal{O}\left(\delta \theta^{3}\right)$. We will see that this approximation is valid within the accuracy of the expansion. With Eq. (16) we get,

$$
\delta \theta=\frac{\frac{3}{2(1-x)} \operatorname{Re} \Delta G^{V J}-\frac{1}{2} \operatorname{Im} \Delta G^{J J}}{W^{-1} V^{-\frac{3}{1-x}} \frac{1+\Delta G^{J \bar{J}}}{V^{2}\left(1+\Delta G^{V V}\right)+\hbar\left(V+\frac{\hbar}{2}\right)}+\frac{1}{2}-\frac{3}{4}\left(\operatorname{Re} \Delta G^{J J}-\Delta G^{J \bar{J}}\right)-\frac{3}{1-x} \operatorname{Im} \Delta G^{V J}+\frac{3(2+x)}{4(1-x)^{2}} \Delta G^{V V}} .
$$

Again, taking the large- $V$ limit, we find

$$
\left.\delta \theta\right|_{\text {large } V}=\frac{\frac{3}{2(1-x)} \operatorname{Re} \Delta G^{V J}-\frac{1}{2} \operatorname{Im} \Delta G^{J J}}{\frac{1}{2}-\frac{3}{4}\left(\operatorname{Re} \Delta G^{J J}-\Delta G^{J \bar{J}}\right)-\frac{3}{1-x} \operatorname{Im} \Delta G^{V J}+\frac{3}{4} \frac{2+x}{(1-x)^{2}} \Delta G^{V V}} .
$$

Thus the expansion is valid for small fractional moments: $\delta \theta$ is small if $\Delta \operatorname{Im} G^{J J}$ and $\Delta \operatorname{Re} G^{V J}$ are small. In this approximation, there is always a solution to the bounce condition (43). It is clear that the bounce cannot disappear as long as this approximation is valid. We are thus not testing the robustness of the bounce itself, but rather some of its properties, such as its energy density.

We can now look at the (square-root of the kinetic) energy at the bounce which from (13) is given by,

$$
\begin{aligned}
\left.H_{Q}\right|_{\text {Bounce }}= & |J| \sin \theta-\frac{W}{|J| \sin \theta} V^{\frac{3}{1-x}} \\
& \times\left\{\frac{1}{2}+\frac{3(2+x)}{4(1-x)^{2}} \Delta G^{V V}-\frac{3 \operatorname{Im} \Delta G^{V J}}{2(1-x) \sin \theta}-\frac{1}{4 \sin ^{2} \theta}\left(\operatorname{Re} \Delta G^{J J}-\Delta G^{J \bar{J}}\right)\right\} .
\end{aligned}
$$

Once again, expanding about $\theta=\pi / 2+\delta \theta$, gives

$$
\begin{aligned}
\left.H_{Q}\right|_{\text {Bounce }}= & |J|-\frac{W V^{\frac{3}{1-x}}}{|J|}\left\{\frac{1}{2}+\frac{3(2+x)}{4(1-x)^{2}} \Delta G^{V V}-\frac{3 \operatorname{Im} \Delta G^{V J}}{2(1-x)}\right. \\
& \left.-\frac{1}{4}\left(\operatorname{Re} \Delta G^{J J}-\Delta G^{J \bar{J}}\right)+\mathcal{O}(\delta \theta)^{2}\right\} .
\end{aligned}
$$

Here it is important to remember our redefinition of the potential: every $W(\phi)$ comes with a $V_{0}$-dependent factor via $f_{0}$ in (9). Using this and the reality condition Eq. (16) one finds that the (square-root of kinetic) energy density at the bounce, again taking the large- $V$ limit, is given by,

$$
\begin{aligned}
\left.\left(\frac{H_{Q}}{\text { vol }}\right)\right|_{\text {Bounce, large } V}= & A-\tilde{W} A\left(\frac{1+\Delta G^{J \bar{J}}}{1+\Delta G^{V V}}\right)\left(\frac{8 \pi \gamma G \tilde{f}_{0}^{2} \tilde{p}^{1+2 x}}{3}\right) \\
& {\left[\frac{1}{2}+\frac{3(2+x)}{4(1-x)^{2}} \Delta G^{V V}-\frac{3 \operatorname{Im} \Delta G^{V J}}{2(1-x)}-\frac{1}{4}\left(\operatorname{Re} \Delta G^{J J}-\Delta G^{J \bar{J}}\right)+\mathcal{O}(\delta \theta)^{2}\right], }
\end{aligned}
$$

where

$$
A \equiv\left\{\frac{|J|}{\operatorname{vol}}\right\}_{\text {large } V}
$$

Using the reality condition Eq. (16) and Eq. (2) we see that this is,

$$
A=\frac{3}{8 \pi G \gamma(1-x)} \tilde{f}_{0}^{-1} \tilde{p}^{-\frac{1+2 x}{2}} \sqrt{\frac{1+\Delta G^{V V}}{1+\Delta G^{J \bar{J}}}}=r(0)
$$

which is just the (square-root of the) energy density at the bounce that we had for the $W=0$ case, Eq. (39). So finally we have that while the bounce is 'close' to the $W=0$ case, its (square-root of the kinetic) energy density is 
given by,

$$
\begin{aligned}
r(W)= & r(0)-\tilde{W} r(0)\left(\frac{8 \pi \gamma G \tilde{f}_{0}^{2} \tilde{p}^{1+2 x}}{3}\right)\left(\frac{1+\Delta G^{J \bar{J}}}{1+\Delta G^{V V}}\right) \\
& {\left[\frac{1}{2}+\frac{3(2+x)}{4(1-x)^{2}} \Delta G^{V V}-\frac{3}{2(1-x)} \operatorname{Im} \Delta G^{V J}+\frac{1}{4}\left(\operatorname{Re} \Delta G^{J J}-\Delta G^{J \bar{J}}\right)\right]+\mathcal{O}(\delta \theta)^{2}, }
\end{aligned}
$$

where

$$
r(W)=\left.\frac{H_{Q}}{\text { vol }}\right|_{\text {Bounce, large } V} .
$$

Just as in the $W=0$ case this is explicitly independent of the fiducial volume.

For the $x=-1 / 2$ case, for instance, this is

$r(W)=r(0)-\tilde{W} r(0)\left(\frac{8 \pi G \gamma \tilde{f}_{0}^{2}}{3}\right)\left(\frac{1+\Delta G^{J \bar{J}}}{1+\Delta G^{V V}}\right)\left[\frac{1}{2}+\frac{1}{2} \Delta G^{V V}-\operatorname{Im} \Delta G^{V J}-\frac{1}{4}\left(\operatorname{Re} \Delta G^{J J}-\Delta G^{J \bar{J}}\right)\right]+\mathcal{O}(\delta \theta)^{2}$,

and the small parameter that we have expanded in is,

$$
\left.\delta \theta\right|_{\text {large } V}=\frac{\Delta \operatorname{Re} G^{V J}-\frac{1}{2} \operatorname{Im} \Delta G^{J J}}{\frac{1}{2}-\frac{3}{4}\left(\Delta \operatorname{Re} G^{J J}-\Delta G^{J \bar{J}}\right)-2 \operatorname{Im} \Delta G^{V J}+\frac{1}{2} \Delta G^{V V}} .
$$

In general, we can say that the (square-root of the kinetic) energy density at the bounce for $x=-1 / 2$, for semiclassical states (i.e. states with fractional moments less than unity), is restricted to the range,

$$
\sqrt{2} r(0)\left[1-\left(\frac{8 \pi G \gamma \tilde{f}_{0}^{2}}{3}\right) \tilde{W}\left(\frac{1+\Delta G^{J \bar{J}}}{1+\Delta G^{V V}}\right) I\right] \geq r(W) \geq \frac{r(0)}{\sqrt{2}}\left[1-\left(\frac{8 \pi G \tilde{f}_{0}^{2}}{3}\right) \tilde{W}\left(\frac{1+\Delta G^{J \bar{J}}}{1+\Delta G^{V V}}\right) I\right]
$$

with

$$
I \equiv \frac{1}{2}+\frac{1}{2} \Delta G^{V V}-\operatorname{Im} \Delta G^{V J}-\frac{1}{4}\left(\operatorname{Re} \Delta G^{J J}-\Delta G^{J \bar{J}}\right)
$$

where we used Eq. (42) and, as before, $r(0)$ is the (square-root of the kinetic) energy density that would have been calculated neglecting the moments entirely.

To decide whether the effect of moments raises or lowers bounce densities, it is more convenient to consider the total rather than kinetic energy density. After all, from the effective Friedmann equation (15) we expect the total density to trigger bounces. With (14), we have the energy density at the bounce given by

$$
\rho_{\text {Bounce }}=\frac{8 \pi G}{3}(1-x)^{2}\left(\frac{H_{Q}}{\operatorname{vol}}\right)^{2}+\tilde{W}=\rho_{\text {crit }} \frac{1+\Delta G^{V V}}{1+\Delta G^{J J}}-D \tilde{W}
$$

with the critical density $\rho_{\text {crit }}$ expected for the free bounce, and (for $x=-1 / 2$ )

$$
D=2 I-1=\Delta G^{V V}-2 \operatorname{Im} \Delta G^{V J}-\frac{1}{2}\left(\operatorname{Re} \Delta G^{J J}-\Delta G^{J \bar{J}}\right) .
$$

Also this correction to the bounce density agrees with the term $\delta_{1}$ from [37] (or $\epsilon_{1}$ from [38]) based on the effective Friedmann equation. By Eq. (31) we have

$$
-\left(\operatorname{Re} \Delta G^{J J}-\Delta G^{J \bar{J}}\right) \geq 0
$$

and $\Delta G^{V V}$ is positive while $\Delta \operatorname{Im} G^{V J}$ does not have definite sign. The sign of $D$ can thus take both values, raising or lowering the bounce density on top of the effect due to the potential. ${ }^{6}$

\footnotetext{
${ }^{6}$ In [43], it was shown that expectation values of a density operator, of the form $\langle$ Energy/volume $\rangle$, are bounded from below by the
} 


\section{DYNAMICAL COHERENT STATES}

Of particular interest are states which saturate the uncertainty bounds Eqs. (24)-(26) and remain saturating them throughout the evolution of the system. Such states are referred to as dynamical coherent states. We will first construct such states for the $W(\phi)=0$ case which is analytically tractable and then discuss the consequences for the rather more complicated case of $W(\phi) \neq 0$.

For a state to initially saturate the uncertainty conditions we require,

$$
\begin{aligned}
2 G^{V V}\left(\operatorname{Re} G^{J J}+G^{J \bar{J}}\right)-4\left(\operatorname{Re} G^{V J}\right)^{2}-\hbar^{2}(\operatorname{Im} J)^{2} & =0, \\
2 G^{V V}\left(-\operatorname{Re} G^{J J}+G^{J \bar{J}}\right)-4\left(\operatorname{Im} G^{V J}\right)^{2}-\hbar^{2}(\operatorname{Re} J)^{2} & =0, \\
\left(G^{J \bar{J}}\right)^{2}-\left(\operatorname{Re} G^{J J}\right)^{2}-\left(\operatorname{Im} G^{J J}\right)^{2}-\hbar^{2}(V+\hbar / 2)^{2} & =0 .
\end{aligned}
$$

These states must also satisfy the reality condition, Eq. (16), which we rewrite here for convenience,

$$
|J|^{2}-\left(V+\frac{1}{2} \hbar\right)^{2}=G^{V V}-G^{J \bar{J}}+\frac{1}{4} \hbar^{2} .
$$

Dynamical coherent states (as with any other state), must also satisfy Eqs. (21)-(22), however these are 'weak' constraints in situations in which third-order moments are not specified, in the sense that they need only be satisfied up to order $(V \hbar)^{3 / 2}$ and hence do not provide sharp constraints on the states.

A state that satisfies these four conditions will initially saturate the uncertainty bounds, however to ensure that it remains saturating them we also require that the derivatives of Eqs. (60)- (62) with respect to $\phi$ be zero. For the $W(\phi)=0$ case one can easily show that the derivative of Eq. (60) with respect to $\phi$ automatically vanishes, while for Eq. (61) and Eq. (62) we require,

$$
2 \operatorname{Re} G^{V J}\left[\operatorname{Re} G^{J J}-G^{J \bar{J}}\right]+2 \operatorname{Im} G^{V J} \operatorname{Im} G^{J J}+\hbar^{2}\left(V+\frac{\hbar}{2}\right) \operatorname{Re} J=0 .
$$

This one constraint is sufficient to ensure that both Eq. (61) and Eq. (62) are independent of $\phi$. This makes the space of dynamical coherent states a hypersurface of co-dimension one in the space of states that instantaneously saturate the uncertainty conditions. Explicit solutions have been found and discussed in [20, 33]. The existence of a large class of coherent states is analogous to the well-known feature of the harmonic oscillator in quantum mechanics, but notice that potentially significant spreading of states is generically possible even for dynamical coherent states [19, 20].

The full parameter space of this system is 9-dimensional, however the five constraints above can be solved to give dynamical coherent states in a 4-dimensional parameter sub-space. A particularly convenient way of solving these constraints is to specify initial data

$$
\left(V, \operatorname{Im} J, \operatorname{Re} G^{J J}, G^{J \bar{J}}\right)
$$

solve Eqs. (60)- -62$)$ as,

$$
\begin{aligned}
\operatorname{Re} G^{V J} & = \pm \sqrt{\frac{1}{2} G^{V V}\left(\operatorname{Re} G^{J J}+G^{J \bar{J}}\right)-\frac{1}{4} \hbar^{2}(\operatorname{Im} J)^{2}} \\
\operatorname{Im} G^{V J} & = \pm \sqrt{\frac{1}{2} G^{V V}\left(-\operatorname{Re} G^{J J}+G^{J \bar{J}}\right)-\frac{1}{4} \hbar^{2}(\operatorname{Re} J)^{2}}=0 \\
\operatorname{Im} G^{J J} & = \pm \sqrt{\left(G^{J \bar{J}}\right)^{2}-\left(\operatorname{Re} G^{J J}\right)^{2}-\hbar^{2}(V+\hbar / 2)^{2}}
\end{aligned}
$$

critical density of the harmonic model even in the presence of a cosmological constant. Our statement is not in conflict with this result because we are considering bounce values for expressions of the form $\langle$ Energy $\rangle /\langle$ volume $\rangle$. Obviously, the two expressions differ by terms depending on the state via its moments, exactly the kind of terms we compute; what we show is that those moments may pull the bounce density below the critical one of the harmonic model. In this context, one should note that [43] refers to a density operator which cannot exist in full loop quantum gravity where only total Hamiltonians, but not energy densities of matter or the gravitational field can be represented as well-defined operators. Expressions such as $\langle$ Energy $\rangle /\langle$ volume $\rangle$ used here, on the other hand, have completely well-defined analogs in the full theory. Thus, our results have a higher degree of robustness than those of [43], even though the mathematical statements of [43] are sharper. 
and then simultaneously solve Eq. (63) and Eq. (64) for $G^{V V}$ and Re $J$. There is an analytic solution to this system, however in practice it is more useful to solve the system for some given initial data i.e. to specify $\left(V, \operatorname{Im} J, \operatorname{Re} G^{J J}, G^{J \bar{J}}\right)$ and solve the resulting simultaneous equations. This is a useful test of the numerical implementation of the system, which we discuss in the following section.

In order to attempt a construction of dynamical coherent states for the $W(\phi) \neq 0$ case, one can in principle follow the same procedure. Clearly the initial states will still have to satisfy the constraints given by Eqs. (60)- (63), however in order for these conditions to be independent of $\phi$ we now need to additionally satisfy,

$$
\begin{array}{r}
2 \frac{\mathrm{d} G^{V V}}{\mathrm{~d} \phi}\left[\operatorname{Re} G^{J J}+G^{J \bar{J}}\right]+2 G^{V V}\left[\frac{\mathrm{dRe} G^{J J}}{\mathrm{~d} \phi}+\frac{\mathrm{d} G^{J \bar{J}}}{\mathrm{~d} \phi}\right]-8 \operatorname{Re} G^{V J} \frac{\mathrm{dRe} G^{V J}}{\mathrm{~d} \phi}-2 \hbar^{2} \operatorname{Im} J \frac{\mathrm{d} \operatorname{Im} J}{\mathrm{~d} \phi}=0, \\
2 \frac{\mathrm{d} G^{V V}}{\mathrm{~d} \phi}\left[-\operatorname{Re} G^{J J}+G^{J \bar{J}}\right]+2 G^{V V}\left[-\frac{\mathrm{d} \operatorname{Re} G^{J J}}{\mathrm{~d} \phi}+\frac{\mathrm{d} G^{J \bar{J}}}{\mathrm{~d} \phi}\right]-8 \operatorname{Im} G^{V J} \frac{\mathrm{dRe} G^{V J}}{\mathrm{~d} \phi}-2 \hbar^{2} \operatorname{Re} J \frac{\mathrm{dRe} J}{\mathrm{~d} \phi}=0, \\
2 G^{J J} \frac{\mathrm{d} G^{J \bar{J}}}{\mathrm{~d} \phi}-2 \operatorname{Im} G^{J J} \frac{\mathrm{dIm} G^{J J}}{\mathrm{~d} \phi}-2 \hbar^{2}\left(V+\frac{\hbar}{2}\right) \frac{\mathrm{d} V}{\mathrm{~d} \phi}=0,
\end{array}
$$

where the derivatives of $G^{a, b}$ are given in Appendix A. These three equations need not be degenerate and certainly are not automatically zero for general states and general $W(\phi)$. They correspond to the single equation Eq. (64) in the $W(\phi)=0$ case. These equations are significantly more difficult to solve. Naively it would appear that we now have seven constraints on the nine-dimensional parameter space that give dynamical coherent states for $W(\phi) \neq 0$. In fact it is easy to see that Eqs. (69)-(71) contain at least four independent conditions on the moments. To see this note that all of the derivatives given in Appendix $\mathrm{A}$ are of the form,

$$
\frac{\mathrm{d} F}{\mathrm{~d} \phi}=F_{0}+W(\phi) F_{1}
$$

where $F_{0}$ and $F_{1}$ have no dependence on $W(\phi)$. It is then clear that Eqs. (69)-(71) contain terms that are independent of $W(\phi)$ and those that are not. For a general potential, these must independently cancel which gives us four conditions. The $W(\phi)$ independent part of Eqs. (69)-(71) gives exactly Eq. (64), while the $W(\phi)$ dependent parts give three additional constraints. Thus we are left with (at most) a one dimensional parameter space that can support dynamical coherent states for a general $W(\phi)$.

Thus far we have been concerned only with the conditions imposed on the moments by the requirement that the uncertainty conditions be saturated and their first derivatives be zero. This alone is not sufficient to fix a dynamical coherent state, since we have to require that all derivatives of the uncertainty conditions vanish. For the $W(\phi)=0$ case, one can explicitly check that the derivative, with respect to $\phi$, of Eq. (64) vanishes provided the uncertainty conditions are saturated (in particular provided Eq. (61) and Eq. (62) are saturated, since Eq. (60) decouples from the system in this case). Thus by inspection Eq. (64) is the only non-trivial condition that needs to be satisfied.

For the $W(\phi) \neq 0$ case we have four constraints to investigate. The $W(\phi)$-independent part of Eqs. (69)-(71) is just Eq. (64) and hence the previous argument holds. For the $W(\phi)$ dependent parts of Eqs. (69)-(71) the situation is less clear, due to the complexity of the evolution equations given in Appendix A. However if any one of the derivatives of any of $W(\phi)$ dependent parts of Eqs. (69)-(71) does not vanish then the parameter space that supports dynamical coherent states reduces to a single point and if any two of the derivatives fail to vanish dynamical coherent states cannot exist.

In addition to the fact that dynamical coherent state may not exist, this reduction in the dimension of the parameter space for such states is also a difficulty for study of the dynamics. Since one can no longer explicitly solve the eight constraints implied by Eqs. (60)- (63) and Eqs. (69)- (71) ${ }^{7}$, small changes in the initial state can lead to radically different trajectories. This is an inherent property of the evolution equations of the states and can thus play a significant role in both analytic and numerical investigations of the dynamics of (nearly) dynamical coherent states (if such states exist). Numerically, difficulties can arise from the large parameter space to be explored when one can no longer stay on the saturation surface. One may reduce the number of initial conditions by starting on the saturation surface, but numerical errors easily lead one into danger of falling onto the wrong side of saturation.

\footnotetext{
7 Recall Eqs. 69)- (71) are four independent equations.
} 


\section{NUMERICAL EVOLUTION OF THE SYSTEM}

The evolution of the moments and their back-reaction on the trajectories of $(J, V)$ can be directly implemented numerically. In particular, because Eq. (27) and the equations in Appendix $\mathrm{A}$ are flows in configuration space (i.e. depend explicitly only on the variables and not their derivatives) the numerical implementation is rather straightforward. Care is however needed to ensure that our numerics are sufficiently accurate so as not to introduce violations of the uncertainty relations, Eqs. (24)-(26), or reality constraints, Eq. (16), (21) and (22), when exact conservation is expected analytically. In the $W \neq 0$ case, as already discussed, exact conservation is not expected since the absence of higher order moments are expected to introduce a fundamental limit to the accuracy of our evolution. Nevertheless, as long as the fractional moments are small, all conditions should be respected.

Another possible point of concern for the general $W(\phi) \neq 0$ case, is that it is no longer guaranteed that $\phi$ will be monotonic. If it is not, then the deparametrization of the system (using $\phi$ as an internal time) will no longer be valid. Such a break down would correspond to a non-unitary evolution of the system and is clearly not physical. This issue is easily avoided, by choosing the $\phi$ coordinate only in regions where it is monotonic and changing coordinates at turn-around points. In our numerical investigation, we avoid this subtlety entirely by focusing our attention on the $W(\phi)=$ const $\neq 0$ cases, for which $\phi$ is monotonic throughout the evolution. Here, we choose $W<0$ so as to get into a bounce regime at larger densities and smaller volume than in the free case.

In practice, fractional moments grow, which can result in the non-conservation of the uncertainty conditions and limiting the length of $\phi$ for which the numerical equations can be evolved. However this is sufficient to examine the bounce, for suitable chosen states. To monitor the growth of higher-order moments which are not included in the evolution equations, we will use plots of the higher-order terms in reality conditions:

$$
\begin{aligned}
V G^{V V}-\operatorname{Re}\left(\bar{J} G^{V J}\right) & =: V^{3} R_{1}, \\
V \operatorname{Re} G^{V J}-\frac{1}{2}\left(\operatorname{Re} J \operatorname{Re} G^{J J}+\operatorname{Im} J \operatorname{Im} G^{J J}+\operatorname{Re} J G^{J \bar{J}}\right) & =: V^{3} R_{2}, \\
V \operatorname{Im} G^{V J}-\frac{1}{2}\left(\operatorname{Re} J \operatorname{Im} G^{J J}-\operatorname{Im} J \operatorname{Re} G^{J J}+\operatorname{Im} J G^{J \bar{J}}\right) & =: V^{3} R_{3} .
\end{aligned}
$$

¿From the general behavior of the moments, we expect $R_{1}, R_{2}$ and $R_{3}$ to be significantly smaller than the fractional second-order moments, e.g. $R_{1} \sim\left(\Delta G^{V V}\right)^{3 / 2} \sim(\hbar / V)^{3 / 2}$. In addition, it is rather difficult to find initial conditions that simultaneously satisfy all of the uncertainty conditions. Thus it is often more convenient to use the uncertainty conditions to fix some of the initial data, by requiring that the state under saturate Eqs. (24)-(26) by some constant i.e. that the state satisfy, for example,

$$
2 \Delta G^{V V}\left(\Delta \operatorname{Re} G^{J J}+\Delta G^{J \bar{J}}\right)-4\left(\Delta \operatorname{Re} G^{V J}\right)^{2}=\hbar^{2} \frac{(\operatorname{Im} J)^{2}}{V^{2}|J|^{2}}+U_{1},
$$

and similarly for the remaining two uncertainty conditions. In general $U_{1}, U_{2}$ and $U_{3}$ depend on $\phi$, however, from Section IV we see that for the $W=0$ case, $U_{1}$ should be a constant, while $U_{2}$ and $U_{3}$ should evolve similarly.

For the numerical calculations we used units in which $\hbar=1$ and $V$ is dimensionless. A typical example for $W(\phi)=0$ is given in Figure 1, which had the following initial conditions:

$$
\left(\begin{array}{ccccccccc}
V & \operatorname{Re} J & \operatorname{Im} J & \Delta G^{V V} & \Delta G^{J \bar{J}} & \Delta \operatorname{Re} G^{V J} & \operatorname{Im} \Delta G^{V J} & \operatorname{Re} \Delta G^{J J} & \operatorname{Im} \Delta G^{J J} \\
35.0 & 24.0 & 25.0 & 0.0331 & 0.0833 & 0.0377 & 0.0294 & 0.0250 & 0.0737
\end{array}\right),
$$

with $x=-1 / 2$. These initial conditions under-saturate the uncertainty conditions, Eq. (24)-(26) and also saturates their derivatives with respect to $\phi$ (Eq. (64)). In addition, one can explicitly check that Eqs. (21)-(22) are satisfied, up to the correct order, throughout the evolution of the system. In the numerical evolutions, we have taken $\hbar=1$, hence one needs to check that Eqs. (21)-(22) are satisfied up to order $\sqrt{\Delta G_{V V}}$, (or other second order moments), since this is the order of the terms neglected.

For this state the bounce occurs at $V\left(\phi_{\text {Bounce }}\right)=25.6582$, with the square-root of the dimensionless kinetic energy density $r(0)=0.974348$. This can be compared to the expected value, given by Eq. (39), of $r(0)=0.974349$, showing the excellent agreement of the numerical implementation with the analytic solutions. In the $W(\phi)=0$ case, the evolution equations given in Appendix $\mathrm{A}$ are exact and hence we can analytically calculate the (square-root of the) energy density at the bounce to arbitrary accuracy (for any given value of $\Delta G_{V V}$ and $\Delta G_{J \bar{J}}$ at the bounce). This is not true for $W(\phi) \neq 0$, since the evolution equations are valid only up to order $\mathcal{O}\left(W^{2}\right)$. However even for the $W=0$ case numerical approximation of the derivatives introduces an error, this can become significant for states that (almost) exactly saturate the uncertainty bounds, where numerical artifacts may lead to unphysical trajectories.

If the saturation of the uncertainty conditions, Eqs. (60)-(62), and their derivatives (Eq. (64) for the $W(\phi)=0$ case) were met exactly then the state would saturate the uncertainty conditions and remain saturating them, through 


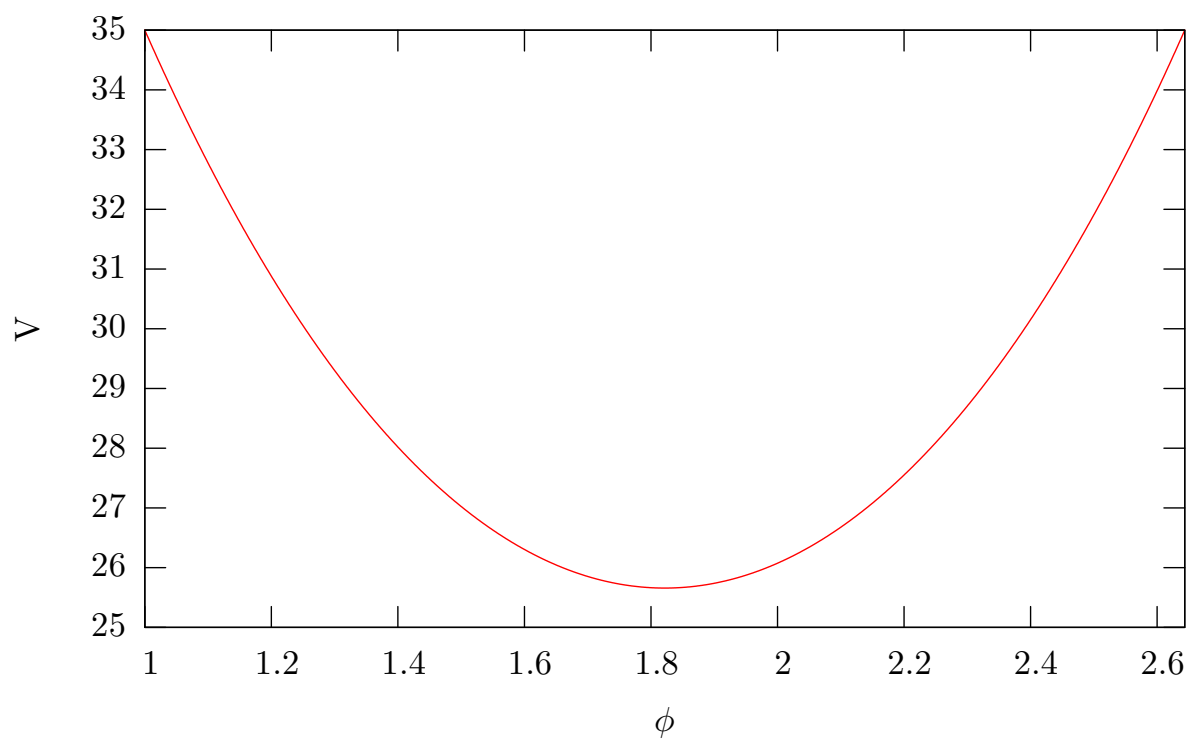

FIG. 1: The trajectory $V(\phi)$, evolved numerically for the initial conditions given by Eq. (777), for the zero potential $(W(\phi)=0)$ case. Note that only the region around the bounce is plotted. This bounce occurs at $V \approx 25.6582$ with a corresponding (squareroot of the) energy density of 0.9743 . Note that for this trajectory, the bounce occurs at $\phi_{\text {Bounce }} \approx 1.821815$.

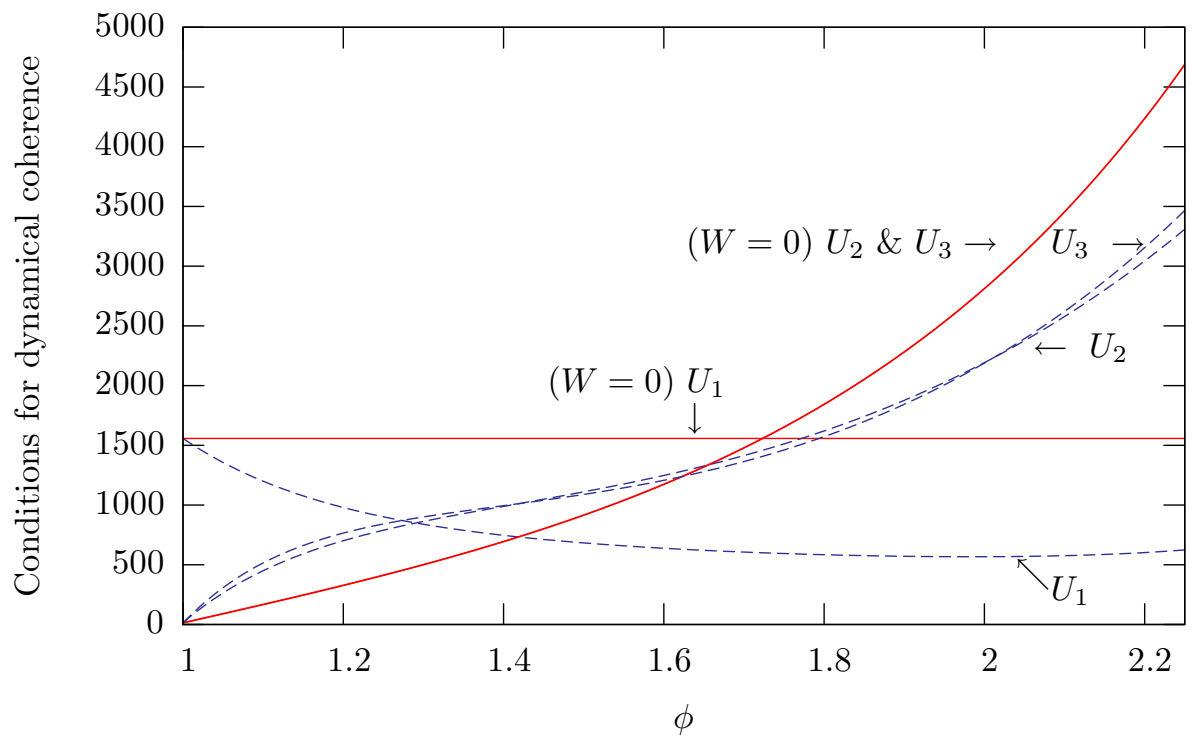

FIG. 2: The system was initialized with a state that both under-saturates the uncertainty conditions, Eq (24)-(26) and ensures that the derivatives with respect to $\phi$ are all positive. Plotted are the values of $U_{1}, U_{2}, 4.0 \times U_{3}$ for the $W(\phi)=0$ and $W(\phi)=-0.05$ cases. Recall that $U_{1}, U_{2}$ and $U_{3}$ positive indicates that the uncertainty conditions are under saturated i.e. physically acceptable. Clearly all the uncertainty conditions are satisfied, in particular at the bounce, which occurs at $\phi \approx 1.8$. Notice that for $W=0, U_{1}$ is essentially constant as expected from the discussion above Eq. (64). Note that the initial conditions are deliberately taken so as to ensure that the system is far from the uncertainty conditions throughout the bounce.

out the evolution in $\phi$. For the state here, the initial data was chosen so that the uncertainty conditions, Eq. (76), were initially under-saturated and the the derivatives of these equations with respect to $\phi$ are positive for both the $W(\phi)=0$ and $W(\phi)=-0.05$ cases. This ensures that, at least initially, the system is evolving towards the undersaturated side of the uncertainty condition hypersurface. Figure 2 shows how the uncertainty constraints evolve for both the $W=0$ and $W=-0.05$ cases.

For the case of $W(\phi)=-0.05$, the trajectory $V(\phi)$ is plotted in Fig. 3, with the initial conditions again given by Eq. (77). In this case the kinetic energy density is found to be higher than the $W(\phi)=0$ case, see Table \ (which 


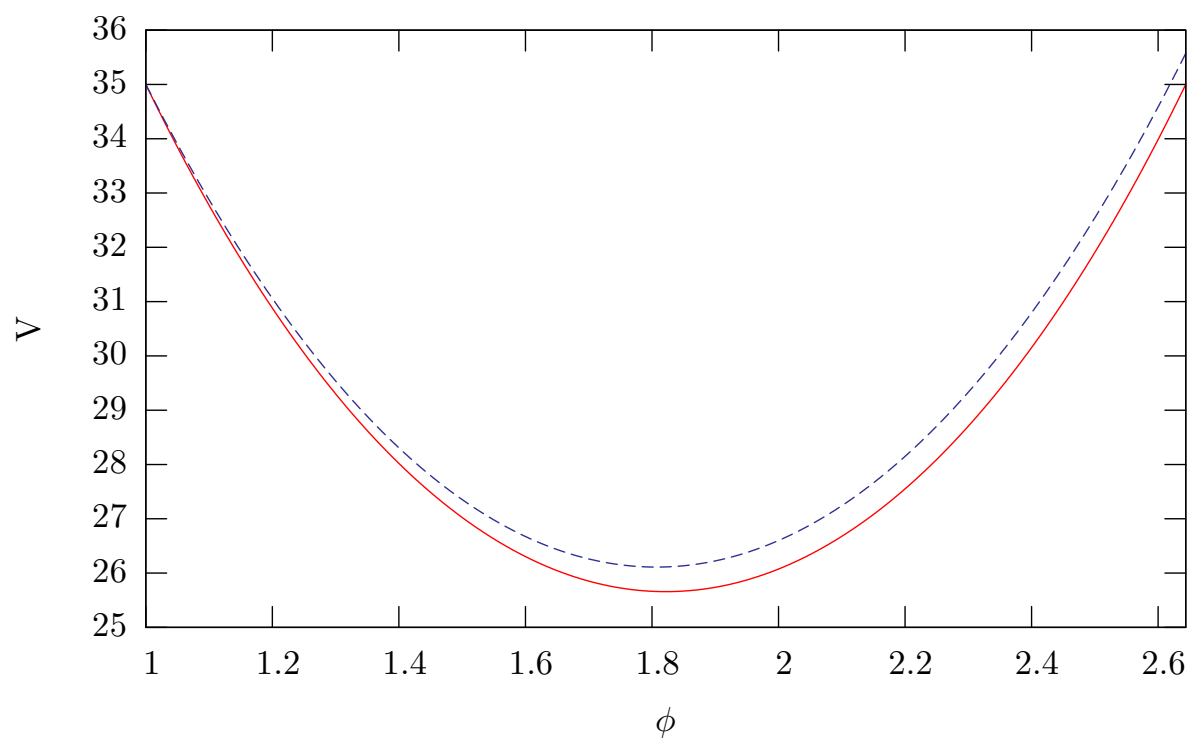

FIG. 3: The trajectory $V(\phi)$, evolved numerically for the initial conditions given by Eq. (77). Again only the bounce region is plotted. The solid curve correspond to the $W(\phi)=0$ case and the dashed curve to the $W(\phi)=-0.05$ case. The bounce for this latter case occurs at $V \approx 25.11053$ with a corresponding square-root energy density of 1.00515 . This is, approximately $3.2 \%$ higher than the $W(\phi)=0$ case, a change mainly (but not solely) attributed to the negative potential energy; see below.

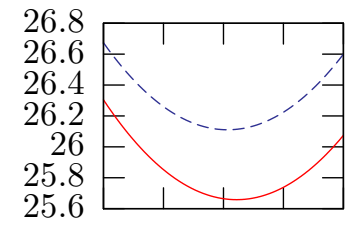

$\begin{array}{llllll}1.6 & 1.7 & 1.8 & 1.9 & 2\end{array}$
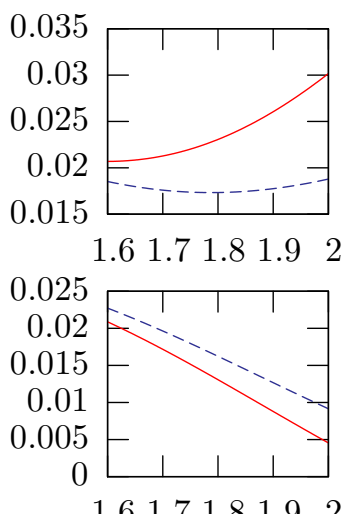
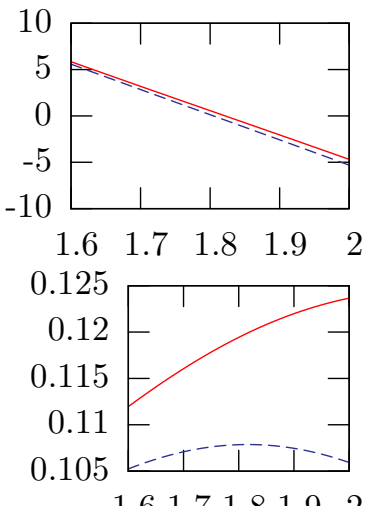

1.61 .71 .81 .92

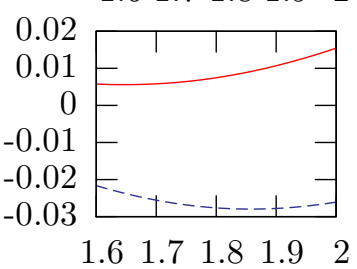

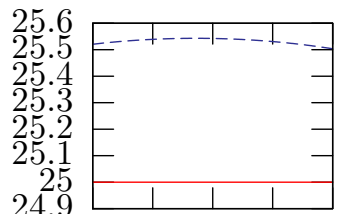

$\begin{array}{llllll}1.6 & 1.7 & 1.8 & 1.9 & 2\end{array}$

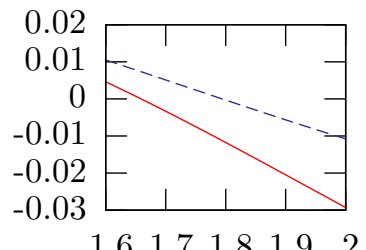

0.0975

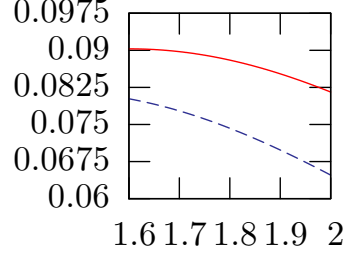

FIG. 4: The trajectories of all the variables over the bounce range, for the initial conditions given in Eq. (77). The solid lines are the $W(\phi)=0$ case and the dashed lines correspond to the $W(\phi)=-0.05$ potential. From top left to bottom right the trajectories are: $V(\phi), \operatorname{Re} J(\phi), \operatorname{Im} J(\phi), \Delta G^{V V}(\phi), \Delta G^{J \bar{J}}(\phi), \Delta \operatorname{Re} G^{V J}(\phi), \operatorname{Im} \Delta G^{V J}(\phi), \Delta \operatorname{Re} G^{J J}(\phi)$ and $\operatorname{Im} \Delta G^{J J}(\phi)$. Notice in particular that for $W(\phi)=0$ we have $\operatorname{Im} J$ a constant, which is expected since for this case we have $\operatorname{Im} J=H_{Q}$ which is conserved and that several of the variables $\left(\operatorname{Re} J(\phi), \Delta G^{J \bar{J}}(\phi)\right.$ etc.) evolve asymmetrically around the bounce point.

\begin{tabular}{|c|c|c|c|}
\hline \hline \multicolumn{2}{|c|}{$W(\phi)$} & 0 & -0.05 \\
\hline$\frac{H_{Q}}{\mathrm{vol}}$ & & 0.97438 & 1.01515 \\
\hline
\end{tabular}

TABLE I: The kinetic energy density of the bounce depends on the magnitude and form of the potential $(W(\phi))$ that is present. The initial conditions are given by Eq. (77) 


\begin{tabular}{|l|c|c|c|}
\hline \hline Estimate & Eq. (48), $\delta \theta^{2} \approx 0.0005$ & numerical \\
\hline$\frac{H_{Q}}{\mathrm{vol}}$ & 1.0038 & 1.0052 \\
\hline
\end{tabular}

TABLE II: The energy density of the bounce for the $W(\phi)=-0.05$ case, calculated using the approximate analytic results Eq. (48) and Eq. (49) compared to the numerical result.

gives the square-root of the energy densities). Using the values of the moments at the bounce, we can evaluate the expected Hamiltonian density from Eqs. (48)-(49) and compare them to the numerical result, see Table In Notice that for this case the evolution equations given in Appendix $\mathrm{A}$ are only valid up to $\mathcal{O}\left(W^{2}\right)=2.5 \times 10^{-3}$ and an accuracy beyond $\sim 3$ significant figures would require the use of higher orders in the expansion. We can see that the approximation made in order to solve Eq. (54) is accurate to, approximately, 0.5\%. It is also worth noting that the moments, as seen in Figure 4 , do not evolve symmetrically around the bounce point. In particular $\operatorname{Re} J, \Delta G^{J \bar{J}}$, $\Delta \operatorname{Re} G^{V J}$, Im $\Delta G^{V J}$ and $\operatorname{Im} \Delta G^{J J}$ all decrease monotonically across the bounce (for this system). This may be significant for deparametrizing the system in order to use one of these variables as an internal time parameter, as suggested in [4].

For the case of constant $W$ as studied here, the effect of varying the moments is small but observable (for the evolutions considered here). In Figure 5 the trajectories $V(\phi)$ for various initial $\Delta G^{J \bar{J}}$ in the range, $\left.\Delta G^{J \bar{J}}\right|_{\text {bounce }}=$ $(0.108,0.189)$. For $W(\phi)=-0.05$ the effect of varying the initial moments is even more pronounced than for $W=0$. Figure 6 shows the dependencies of the (square-root of the) energy densities at the bounce on the initial value of $\Delta G^{J \bar{J}}$, for both the $W(\phi)=0$ and $W(\phi)=-0.05$ cases. In the cases examined here, $\Delta G^{J \bar{J}}$ is approximately proportional to $\Delta G^{V V}$ at the bounce. Hence, by Eq. (40), we would not expect there to be a significant dependence on $\Delta G^{J \bar{J}}$ for the $W=0$ case, which is what is found. For the $W \neq 0$ cases however there is a dependence, due to the terms proportional to $W$ in Eq. (48).

In Section $[\nabla]$ we described how to explicitly construct dynamical coherent states for the $W(\phi)=0.0$ case. Given the initial data,

$$
\left(V, \operatorname{Im} J, G^{J \bar{J}}, \operatorname{Re} G^{J J}\right)=(105.0,100.0,125.0,-1.0),
$$

we can solve Eq. (76), with $U_{1}=U_{2}=U_{3}=0.0$ (which is required if the state is to be dynamically coherent) and Eq. (64) to find (for example)

$$
\left(G_{V V}, \operatorname{Re} J, \operatorname{Re} G_{V J}, \operatorname{Im} G_{V J}, \operatorname{Im} G_{J J}\right) \approx(82.52970,32.985295,51.15507,70.195184,67.035438) .
$$

We have checked carefully that for this case (and others that are expected to be dynamically coherent) the uncertainty relations are indeed conserved up to numerical rounding errors (which can be made negligibly small by employing a suitably accurate discretization scheme). This provides a strong test of the accuracy of our numerical implementation

Finally as an example of moments which evolve to break the uncertainty relations (in this particular case Eq. (61)) showing the breakdown of the approximation, in Fig. 7 we plot the evolution of $U_{1}, U_{2}$ and $U_{3}$ for the initial conditions

$$
\left(\begin{array}{ccccccccc}
V & \operatorname{Re} J & \operatorname{Im} J & \Delta G^{V V} & \Delta G^{J \bar{J}} & \Delta \operatorname{Re} G^{V J} & \operatorname{Im} \Delta G^{V J} & \operatorname{Re} \Delta G^{J J} & \operatorname{Im} \Delta G^{J J} \\
35.0 & 24.0 & 25.0 & 0.0331 & 0.09159 & 0.04267 & 0.02363 & 0.0250 & 0.0737
\end{array}\right) .
$$

This initial state initially undersaturates all the uncertainty conditions $\left(U_{1} \approx 18.1, U_{2} \approx 2623.9\right.$ and $U_{3} \approx 2103.6$ at $\phi=1$ ), but dynamically evolves to break the uncertainty bounds. As one can see from Fig. 7 the moments are no longer small for this state $\left(\Delta G^{J \bar{J}}\right.$ and $\operatorname{Im} \Delta G^{J J}$ are larger than $\left.10 \%\right)$, which indicates that the contributions to the dynamics from higher order moments can no longer be neglected. Note that even for this case, there is a bounce at $\phi \approx 1.82$, with square root of the energy density being $\rho_{\text {bounce }} \approx 0.97435$ and despite the fact that the evolution of this state has significant contribution from higher order moments, this value is within $0.72 \%$ of that predicted by Eq. (39). Thus we see that our estimates for the (square root of the) energy density at the bounce are well approximated using only the first order moment, at least while the dynamics of the state do not breach the uncertainty conditions Eq. (60) - (62) . Once again we emphasize that this breaching of the uncertainty conditions is not an artifact of the numerical implementation, rather it is a genuine feature of the dynamics, due to the truncation at second order in the moments. 


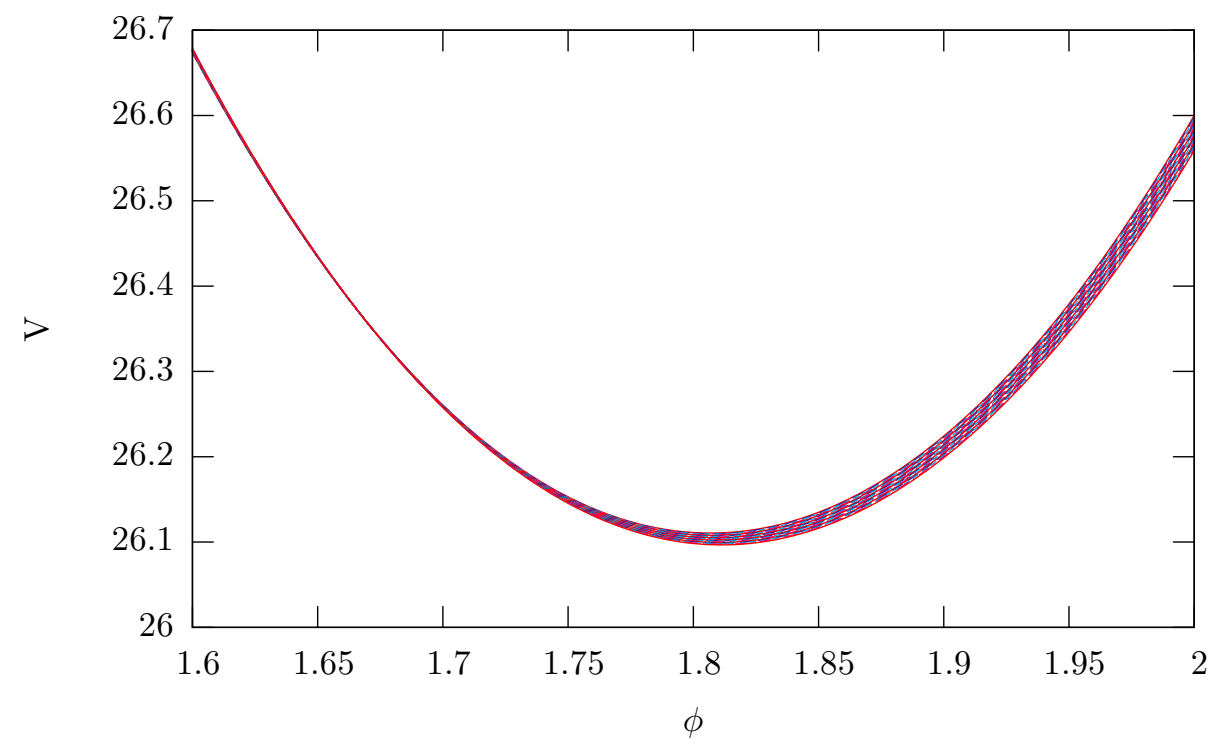

FIG. 5: The trajectory $V(\phi)$, evolved numerically for the initial conditions given by Eq. (777). Again only the bounce region is plotted. In this case, the potential was set to be -0.05 . The various plots correspond to different initial values of $\Delta G_{J} \bar{J}$ in the range $\left.\Delta G^{J \bar{J}}\right|_{\text {bounce }}=0.107779 \rightarrow 0.18926\left(\left.G_{J \bar{J}}\right|_{\text {initial }}=100.0 \rightarrow 140.0\right.$, in steps of $\left.\delta G_{J \bar{J}}=1.0\right)$. The sensitivity to variations of the moments is significantly increased after the bounce, an observation extending the conclusions of [19, 20].

\section{CONCLUSIONS}

Numerical investigations in loop quantum cosmology have so far only considered semi-classical states of Gaussian type, peaked around some particular value [42, 45, 46]. The expectation value of such states has been shown to closely follow the classical trajectories, except at very high densities where quantum gravity effects, but primarily those of quantum geometry, produce a bounce. Implicit in these analyzes is the idea that a general semi-classical state is well characterized by its expectation values and small spreads alone and that the evolution of the spread and other moments does not affect the evolution of the peak. Here we have considered a moment expansion of a state around such exactly solvable Gaussian states as a way of probing the consequences of relaxing this assumption. In principle one should consider all orders of the moment expansion, however in order to make the system tractable, we have truncated the series at second order. Already this expands the configuration space from 4-dimensional $((V, \phi)$ and their momenta) to 18-dimensional (given by the eight independent variables in Eq. (28), $\phi$ and their momenta).

The inclusion of moments introduces sets of non-trivial uncertainty relations that must be satisfied by the state at all points along its trajectory. Dynamical coherent states are those for which all the uncertainty relations are exactly saturated, throughout the state's evolution. We have shown that the conditions necessary to ensure a state is dynamical coherent, effectively reduces the dimension of the state's phase space i.e. the conditions form a constraint surface on which the states, and their trajectories, lie. For the zero potential case, there is only one condition that needs to be met to ensure that a state which initially saturates the uncertainty bounds will be a dynamical coherent state (i.e. will continue to saturate the bounds). For a system with a non-zero potential one has four independent constraints (in addition to the uncertainty bounds) which need to be met, just to ensure that the first derivatives of the uncertainty relations vanish. While requiring that the higher derivatives also vanish is likely to introduce even more constraints. This makes it extremely difficult (and probably impossible) to find exact dynamical coherent states for systems with a non-zero scalar field potential. While conceptually this may be a problem, especially for considering evolution from the infinite past $(\phi \rightarrow-\infty)$ or cyclic models, in practice we are used to dealing with quantum states that are only approximately dynamically coherent, such as particle wave-packets in standard quantum mechanics. Provided the states are approximately dynamically coherent for a sufficiently long period of time $(\phi)$ they can, for all practical purposes, be considered semi-classical.

We have investigated the consequences of the moments on the presence of a bounce, for states that remain semiclassical (in the sense that all their moments are small compared to the expectation values and that the states obey the uncertainty conditions). In all cases the bounce was found to be present and the energy density was changed only marginally from the standard case (i.e. the case in which the moments are neglected). Thus we found that for 


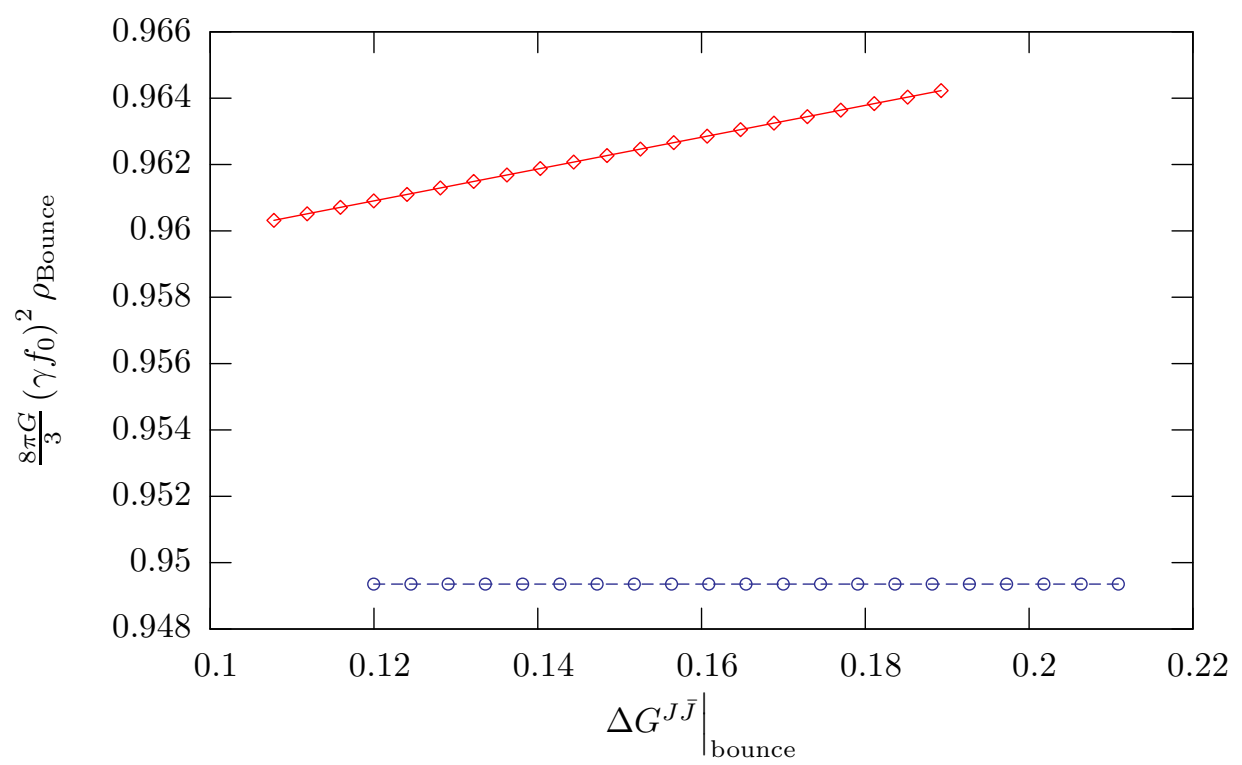

FIG. 6: The energy density of the bounce, given by Eq. (57), for the $W=0$ and $W=-0.05$ cases as a function of $\Delta G^{J \bar{J}}$, with the remaining initial conditions being given by Eq. (77). The $W=0$ case (lower line) is approximately independent of $\Delta G^{J \bar{J}}$ because at the bounce $\Delta G^{J \bar{J}} \sim \Delta G^{V V}$, for the system under consideration, however for $W \neq 0$ (upper line) there is a (small) dependence on $G^{J \bar{J}}$. Note that both plots correspond to the same initial range of $G^{J \bar{J}}=100 \rightarrow 140$. The non-vanishing slope shows that effects on the bounce density are not just due to the non-vanishing potential.
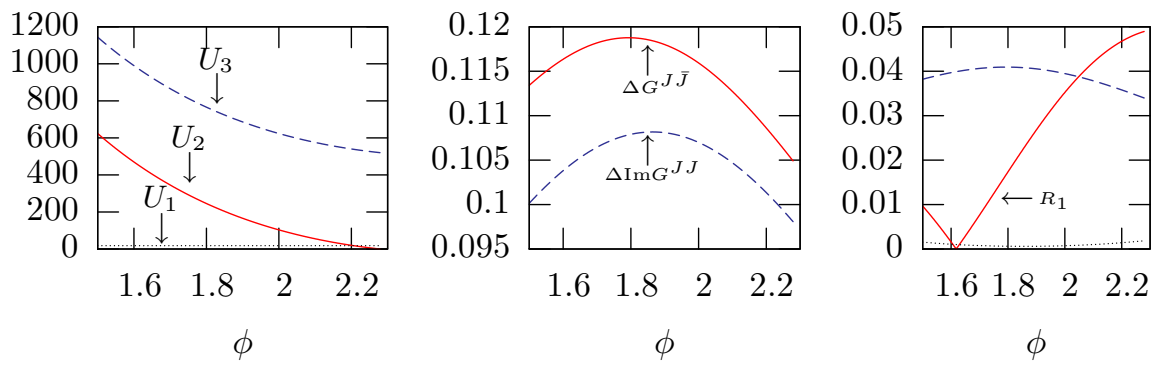

FIG. 7: The left hand plot gives the values of the uncertainty conditions, during the evolution, the center one plots the corresponding values of $\Delta G^{J \bar{J}}$ and $\operatorname{Im} \Delta G^{J J}$. The right hand plot shows $R_{1}$, the order of the terms neglected in Eqs. (21)-(22) compared to the fractional moments $\left(\Delta G^{J \bar{J}}\right)^{3 / 2}$ (the larger values) and $\left(\Delta G^{V V}\right)^{3 / 2}$ (which happens to be much smaller in this evolution). For this evolution all the remaining moments and correlations remain small (less than $\approx 2 \%$ ) and the bounce occurs at $\phi_{\text {bounce }} \approx 1.82$. The increase of $R_{1}$ toward the end of the evolution indicates that neglecting the third order moments is no longer a good approximation in the region in which the uncertainty conditions (in this case $U_{2}$ ) are breached.

the (large number of) cases investigated, the bounce occurs at approximately the same density, a feature not at all unexpected due to the setup of our approximations. The fact that there were small deviations from the standard bound is however significant, especially since we show that it is possible for the bounce density to be sometimes larger than the standard case. We restricted ourselves to considering only states that remained highly semi-classical before, after and during the bounce and still found that the moments noticeably back-react on the trajectories of the expectation values. It may be that there are states which are semi-classical at large scales, but which become dominated by the evolution of their moments at small scales (i.e. become highly non-semi-classical) and for such states the presence of a bounce is not guaranteed by current results in the literature. 


\section{Acknowledgments}

This work was supported in part by NSF grants PHY0748336, PHY0854743, The George A. and Margaret M. Downsbrough Endowment and the Eberly research funds of Penn State. DM is supported by STFC, and RT thanks the hospitality of Tokyo University of Science, University of Tokyo and Rikkyo University, where this work was finalized during a visit supported by a Royal Society bilateral grant.

\section{Appendix A: Evolution equations}

The evolution of the variables $V, J$ and their moments with respect to the scalar field $\phi$ are [40]:

$$
\begin{aligned}
& \frac{\mathrm{d} V}{\mathrm{~d} \phi}=-\frac{J+\bar{J}}{2}+\frac{J+\bar{J}}{(J-\bar{J})^{2}} V^{3 /(1-x)} W(\phi)+3 \frac{J+\bar{J}}{(J-\bar{J})^{4}} V^{3 /(1-x)}\left(G^{J J}+G^{\bar{J} \bar{J}}-2 G^{J \bar{J}}\right) W(\phi) \\
& -\frac{6}{1-x} \frac{J+\bar{J}}{(J-\bar{J})^{3}} V^{(2+x) /(1-x)}\left(G^{V J}-G^{V \bar{J}}\right) W(\phi)+\frac{3}{2} \frac{2+x}{(1-x)^{2}} \frac{J+\bar{J}}{(J-\bar{J})^{2}} V^{(1+2 x) /(1-x)} G^{V V} W(\phi) \\
& -\frac{2 V^{3 /(1-x)}}{(J-\bar{J})^{3}}\left(G^{J J}-G^{\bar{J} \bar{J}}\right) W(\phi)+\frac{3}{1-x} \frac{V^{(2+x) /(1-x)}}{(J-\bar{J})^{2}}\left(G^{V J}+G^{V \bar{J}}\right) W(\phi) \\
& \frac{\mathrm{d} J}{\mathrm{~d} \phi}=-(V+\hbar / 2)+\left(\frac{3}{1-x} \frac{V^{(2+x) /(1-x)} J}{J-\bar{J}}+\frac{2 V^{3 /(1-x)}(V+\hbar / 2)}{(J-\bar{J})^{2}}\right) W(\phi) \\
& +\left(\frac{3}{1-x} \frac{V^{(2+x) /(1-x)} J}{(J-\bar{J})^{3}}+\frac{6 V^{3 /(1-x)}(V+\hbar / 2)}{(J-\bar{J})^{4}}\right)\left(G^{J J}+G^{\bar{J} \bar{J}}-2 G^{J \bar{J}}\right) W(\phi) \\
& -3\left(\frac{2+x}{(1-x)^{2}} \frac{V^{(1+2 x) /(1-x)} J}{(J-\bar{J})^{2}}+\frac{4}{1-x} \frac{V^{(2+x) /(1-x)}(V+\hbar / 2)}{(J-\bar{J})^{3}}\right)\left(G^{V J}-G^{V \bar{J}}\right) W(\phi) \\
& +\frac{3}{2}\left(\frac{(2+x)(1+2 x)}{(1-x)^{3}} \frac{V^{3 x /(1-x)} J}{J-\bar{J}}+\frac{2(2+x)}{(1-x)^{2}} \frac{V^{(1+2 x) /(1-x)}(V+\hbar / 2)}{(J-\bar{J})^{2}}\right) G^{V V} W(\phi) \\
& +4 \frac{V^{3 /(1-x)}}{(J-\bar{J})^{3}}\left(G^{V \bar{J}}-G^{V J}\right) W(\phi) \\
& -\frac{3}{1-x} \frac{V^{(2+x) /(1-x)}}{(J-\bar{J})^{2}}\left(G^{J J}-G^{J \bar{J}}-2 G^{V V}\right) W(\phi)+3 \frac{2+x}{(1-x)^{2}} \frac{V^{(1+2 x) /(1-x)}}{J-\bar{J}} G^{V J} W(\phi) \\
& \frac{\mathrm{d} G^{V V}}{\mathrm{~d} \phi}=-\left(G^{V J}+G^{V \bar{J}}\right)+\frac{2 V^{3 /(1-x)}}{(J-\bar{J})^{2}}\left(G^{V J}+G^{V \bar{J}}\right) W(\phi) \\
& -\frac{4 V^{3 /(1-x)}}{(J-\bar{J})^{3}}\left(J G^{V J}-\bar{J} G^{V \bar{J}}-J G^{V \bar{J}}+\bar{J} G^{V J}\right) W(\phi) \\
& +\frac{6}{1-x} \frac{V^{(2+x) /(1-x)}(J+\bar{J})}{(J-\bar{J})^{2}} G^{V V} W(\phi)-\frac{\hbar^{2}}{2(1-x)} \frac{V^{(2+x) /(1-x)}(J+\bar{J})}{(J-\bar{J})^{2}} W(\phi) \\
& =-\left(1 / W(\phi)-\frac{2 V^{3 /(1-x)}}{(J-\bar{J})^{2}}\right)\left(G^{V J}+G^{V \bar{J}}\right) W(\phi)-\frac{4 V^{3 /(1-x)}(J+\bar{J})}{(J-\bar{J})^{3}}\left(G^{V J}-G^{V \bar{J}}\right) W(\phi) \\
& +\frac{6}{1-x} \frac{V^{(2+x) /(1-x)}(J+\bar{J})}{(J-\bar{J})^{2}} G^{V V} W(\phi)-\frac{\hbar^{2}}{2(1-x)} \frac{V^{(2+x) /(1-x)}(J+\bar{J})}{(J-\bar{J})^{2}} W(\phi) \\
& \frac{\mathrm{d} G^{J \bar{J}}}{\mathrm{~d} \phi}=-\left(G^{V J}+G^{V \bar{J}}\right)+\frac{2 V^{3 /(1-x)}}{(J-\bar{J})^{2}}\left(G^{V J}+G^{V \bar{J}}\right) W(\phi)-\frac{4 V^{3 /(1-x)}(V+\hbar / 2)}{(J-\bar{J})^{3}}\left(G^{J J}-G^{\bar{J} \bar{J}}\right) W(\phi) \\
& -\frac{3}{1-x} \frac{V^{(2+x) /(1-x)}}{(J-\bar{J})^{2}}\left((J+\bar{J}) G^{J \bar{J}}-(2 V+\hbar)\left(G^{V J}+G^{V \bar{J}}\right)-\bar{J} G^{J J}-J G^{\bar{J} \bar{J}}\right.
\end{aligned}
$$




$$
\begin{aligned}
& \left.+\frac{1}{6} \hbar^{2}(J+\bar{J})\right) W(\phi)+\frac{3(2+x)}{(1-x)^{2}} \frac{V^{(1+2 x) /(1-x)}}{J-\bar{J}}\left(J G^{V \bar{J}}-\bar{J} G^{V J}\right) W(\phi) \\
& =-\left(G^{V J}+G^{V \bar{J}}\right) \\
& +\left(\frac{3}{2} \frac{2+x}{(1-x)^{2}} V^{(1+2 x) /(1-x)}+\frac{2 V^{(2+x) /(1-x)}(V+3(V+\hbar / 2) /(1-x))}{(J-\bar{J})^{2}}\right)\left(G^{V J}+G^{V \bar{J}}\right) W(\phi) \\
& -\frac{3}{2} \frac{2+x}{(1-x)^{2}} \frac{V^{(1+2 x) /(1-x)}(J+\bar{J})}{J-\bar{J}}\left(G^{V J}-G^{V \bar{J}}\right) W(\phi) \\
& -\frac{3}{2} \frac{V^{(2+x) /(1-x)}}{J-\bar{J}}\left(\frac{1}{1-x}+\frac{8}{3} \frac{V(V+\hbar / 2)}{(J-\bar{J})^{2}}\right)\left(G^{J J}-G^{\bar{J} \bar{J}}\right) W(\phi) \\
& +\frac{3}{2(1-x)} \frac{V^{(2+x) /(1-x)}(J+\bar{J})}{(J-\bar{J})^{2}}\left(G^{J J}+G^{\bar{J} \bar{J}}-2 G^{J \bar{J}}\right) W(\phi)-\frac{\hbar^{2}}{2(1-x)} \frac{V^{(2+x) /(1-x)}(J+\bar{J})}{(J-\bar{J})^{2}} W(\phi) \\
& \frac{\mathrm{d} G^{V J}}{\mathrm{~d} \phi}=-\frac{1}{2}\left(G^{J J}+G^{J \bar{J}}+2 G^{V V}\right)+\frac{V^{3 /(1-x)}}{(J-\bar{J})^{2}}\left(G^{J J}+G^{J \bar{J}}+2 G^{V V}\right) W(\phi)+\frac{3}{1-x} \frac{V^{(2+x) /(1-x)}}{J-\bar{J}} G^{V J} W^{(\phi)}(\mathrm{A} \\
& +\frac{V^{3 /(1-x)} W(\phi)}{(J-\bar{J})^{3}}\left(-2(J+\bar{J}) G^{J J}-2 J G^{\bar{J} \bar{J}}+2(V+\hbar / 2)\left(4 G^{V \bar{J}}-2 G^{V J}\right)+2 J G^{J \bar{J}}\right. \\
& \left.-\hbar^{2} \bar{J}+\frac{1}{3} \hbar^{2} J\right)+\frac{3}{1-x} \frac{V^{(2+x) /(1-x)} W(\phi)}{(J-\bar{J})^{2}}\left(6(V+\hbar / 2) G^{V V}-\bar{J} G^{V J}-J G^{V \bar{J}}+\frac{1}{2} \hbar^{2} V+\frac{1}{4} \hbar^{3}\right) \\
& +3 \frac{2+x}{(1-x)^{2}} \frac{V^{(1+2 x) /(1-x)} W(\phi)}{J-\bar{J}}\left(J G^{V V}-\frac{1}{12} \hbar^{2} J\right) \\
& =\overline{\dot{G}^{V \bar{J}}} \\
& \begin{aligned}
\frac{\mathrm{d} G^{J J}}{\mathrm{~d} \phi}= & -2 G^{V J}+4 \frac{V^{3 /(1-x)}}{(J-\bar{J})^{2}} G^{V J} W(\phi)+\frac{6}{1-x} \frac{V^{(2+x) /(1-x)}}{J-\bar{J}} G^{J J} W(\phi) \\
& +\frac{V^{3 /(1-x)} W(\phi)}{(J-\bar{J})^{3}}\left(8(V+\hbar / 2)\left(2 G^{V V}+G^{J \bar{J}}-G^{J J}\right)-8 J G^{V \bar{J}}-8 \bar{J} G^{V J}+4 \hbar^{2} V+2 \hbar^{3}\right) \\
& -\frac{3}{1-x} \frac{V^{(2+x) /(1-x)} W(\phi)}{(J-\bar{J})^{2}}\left(2 J G^{J J}-8(V+\hbar / 2) G^{V J}+2 \bar{J} G^{J J}+\hbar^{2} J\right)+\frac{6(2+x)}{(1-x)^{2}} \frac{V^{(1+2 x) /(1-x)}}{J-\bar{J}} \\
= & \frac{\dot{G}^{\bar{J} \bar{J}}}{}
\end{aligned}
\end{aligned}
$$

Equations of motion for real and imaginary parts of $J, G^{V J}$ and $G^{J J}$ are:

$$
\begin{aligned}
\frac{\mathrm{d}}{\mathrm{d} \phi} \operatorname{Re} J= & -\left(V+\frac{\hbar}{2}\right)+\frac{1}{2}\left(\frac{3}{1-x} V^{(2+x) /(1-x)}-\frac{V^{(4-x) /(1-x)}}{(\operatorname{Im} J)^{2}}\right) W(\phi) \\
& +\frac{3}{4} \frac{V^{(4-x) /(1-x)}}{(\operatorname{Im} J)^{4}}\left(\operatorname{Re} G^{J J}-G^{J \bar{J}}\right) W(\phi) \\
& +\left(\frac{3}{(1-x)}-1\right) \frac{V^{3 /(1-x)}}{(\operatorname{Im} J)^{3}} \operatorname{Im} G^{V J} W(\phi) \\
& +\frac{3}{2}\left(\frac{(2+x)(1+2 x)}{2(1-x)^{3}} V^{3 x /(1-x)}-\frac{4-x}{2(1-x)^{2}} \frac{V^{(2+x) /(1-x)}}{(\operatorname{Im} J)^{2}}\right) G^{V V} W(\phi) \\
& +\frac{\hbar}{2} \frac{W(\phi)}{(\operatorname{Im} J)^{2}} V^{3 /(1-x)}\left[\frac{-1}{2}+\frac{3}{4(\operatorname{Im} J)^{2}}\left(\operatorname{Re} G^{J J}-G^{J J}\right)\right. \\
& \left.+\frac{3}{(1-x) \operatorname{Im} J} \operatorname{Im} G^{V J} V^{-1}-\frac{3(2+x)}{4(1-x)^{2}} G^{V V} V^{-2}\right], \\
\frac{\mathrm{d}}{\mathrm{d} \phi} \operatorname{Im} J=-\frac{3(1-x)}{2(1-x)} & \frac{V^{(2+x) /(1-x)} \operatorname{Re} J}{\operatorname{Im} J} W(\phi)-\frac{3}{4} \frac{(2+x)(1+2 x)}{(1-x)^{3}} \frac{V^{3 x /(1-x)} \operatorname{Re} J}{\operatorname{Im} J} G^{V V} W(\phi)
\end{aligned}
$$




$$
\begin{aligned}
& +\frac{3}{4(1-x)} \frac{V^{(2+x) /(1-x)} \operatorname{Re} J}{(\operatorname{Im} J)^{3}}\left(\operatorname{Re} G^{J J}-G^{J \bar{J}}\right) W(\phi)+\frac{3}{4(1-x)} \frac{V^{(2+x) /(1-x)}}{(\operatorname{Im} J)^{2}} \operatorname{Im} G^{J J} W(\phi) \\
& +\frac{3}{2} \frac{2+x}{(1-x)^{2}} \frac{V^{(1+2 x) /(1-x)} \operatorname{Re} J}{(\operatorname{Im} J)^{2}} \operatorname{Im} G^{V J} W(\phi)-\frac{3}{2} \frac{2+x}{(1-x)^{2}} \frac{V^{(1+2 x) /(1-x)}}{\operatorname{Im} J} \operatorname{Re} G^{V J} W(\phi) \\
& \frac{\mathrm{d}}{\mathrm{d} \phi} \operatorname{Re} G^{V J}=-\frac{1}{2}\left(\operatorname{Re} G^{J J}+G^{J \bar{J}}+2 G^{V V}\right) \\
& -\frac{1}{2} \frac{V^{3 /(1-x)}}{(\operatorname{Im} J)^{2}}\left(G^{J \bar{J}}+\frac{10-x}{1-x} G^{V V}\right) W(\phi)+\frac{3}{2} \frac{2+x}{(1-x)^{2}} V^{(1+2 x) /(1-x)} G^{V V} W(\phi) \\
& +\left(\frac{3}{2(1-x)} \frac{V^{(2+x) /(1-x)}}{\operatorname{Im} J}+\frac{3}{2} \frac{V^{(4-x) /(1-x)}}{(\operatorname{Im} J)^{3}}\right) \operatorname{Im} G^{V J} W(\phi) \\
& +\frac{3}{2(1-x)} \frac{V^{(2+x) /(1-x)} \operatorname{Re} J}{(\operatorname{Im} J)^{2}} \operatorname{Re} G^{V J} W(\phi)+\frac{1}{4} \frac{V^{3 /(1-x)} \operatorname{Re} J}{(\operatorname{Im} J)^{3}} \operatorname{Im} G^{J J} W(\phi) \\
& +\frac{\hbar}{2} \frac{W(\phi) V^{3 /(1-x)}}{\operatorname{Im} J}\left[\frac{3}{2} \frac{\operatorname{Im} G^{V J}}{(\operatorname{Im} J)^{2}}-\frac{\hbar}{2}\left(\frac{2}{3 \operatorname{Im} J}+\frac{2+x}{2(1-x)^{2}} V^{-2} \operatorname{Re} J\right)\right. \\
& \left.-\frac{3}{1-x} \frac{V^{-1}}{\operatorname{Im} J}\left(G^{V V}+\frac{\hbar}{4}\left(V+\frac{\hbar}{2}\right)\right)\right] \text {, } \\
& \frac{\mathrm{d}}{\mathrm{d} \phi} \operatorname{Im} G^{V J}=-\frac{1}{2} \operatorname{Im} G^{J J}-\frac{1}{2} \frac{V^{(2+x) /(1-x)}}{\operatorname{Im} J}\left(\frac{3}{1-x}-\frac{V^{2}}{(\operatorname{Im} J)^{2}}\right) \operatorname{Re} G^{V J} W(\phi) \\
& -\frac{3}{2} \frac{2+x}{(1-x)^{2}} \frac{V^{(1+2 x) /(1-x)} \operatorname{Re} J}{\operatorname{Im} J} G^{V V} W(\phi)+\frac{1}{4} \frac{V^{3 /(1-x)} \operatorname{Re} J}{(\operatorname{Im} J)^{3}} G^{J \bar{J}} W(\phi) \\
& -\frac{1}{4} \frac{V^{3 /(1-x)}}{(\operatorname{Im} J)^{3}}\left(3 \operatorname{Re} J \operatorname{Re} G^{J J}+\operatorname{Im} J \operatorname{Im} G^{J J}\right) W(\phi) \\
& +\frac{\hbar}{2} W(\phi) V^{3 /(1-x)}\left[\frac{\operatorname{Re} G^{V J}}{2(\operatorname{Im} J)^{3}}-\frac{\hbar}{2}\left(\frac{\operatorname{Re} J}{3(\operatorname{Im} J)^{3}}-\frac{2+x}{16(1-x)^{2}} \frac{V^{-2} \operatorname{Re} J}{\operatorname{Im} J}\right)\right] \\
& \frac{\mathrm{d}}{\mathrm{d} \phi} \operatorname{Re} G^{J J}=-2 \operatorname{Re} G^{V J}+\left(3 \frac{2+x}{(1-x)^{2}} V^{(1+2 x) /(1-x)}+\frac{7-x}{1-x} \frac{V^{3 /(1-x)}}{(\operatorname{Im} J)^{2}}\right) \operatorname{Re} G^{V J} W(\phi) \\
& +3 \frac{2+x}{(1-x)^{2}} \frac{V^{(1+2 x) /(1-x)} \operatorname{Re} J}{\operatorname{Im} J} \operatorname{Im} G^{V J} W(\phi) \\
& +\frac{3}{1-x} \frac{V^{(2+x) /(1-x)} \operatorname{Re} J}{\operatorname{Im} J^{2}} \operatorname{Re} G^{J J} W(\phi) \\
& +\frac{V^{(4-x) /(1-x)}}{(\operatorname{Im} J)^{3}}\left(1+\frac{3}{(1-x)} \frac{\left(\operatorname{Im} J^{2}\right)}{V^{2}}\right) \operatorname{Im} G^{J J} W(\phi) \\
& +\frac{\hbar}{2} \frac{W(\phi) V^{3 /(1-x)}}{(\operatorname{Im} J)^{2}}\left[\frac{\operatorname{Im} G^{J J}}{\operatorname{Im} J}+\frac{12}{1-x} V^{-1}\left(2 \operatorname{Re} G^{V J}-\frac{\hbar}{2} \operatorname{Re} J\right)\right], \\
& \frac{\mathrm{d}}{\mathrm{d} \phi} \operatorname{Im} G^{J J}=-2 \operatorname{Im} G^{V J}-\left(\frac{5+x}{1-x} \frac{V^{3 /(1-x)}}{(\operatorname{Im} J)^{2}}+\frac{6(2+x)}{2(1-x)^{2}} V^{(1+2 x) /(1-x)}\right) \operatorname{Im} G^{V J} W(\phi) \\
& -\left(3 \frac{2+x}{(1-x)^{2}}+2 \frac{V}{(\operatorname{Im} J)^{2}}\right) \frac{V^{(1+2 x) /(1-x)} \operatorname{Re} J}{\operatorname{Im} J} \operatorname{Re} G^{V J} W(\phi) \\
& +\frac{V^{(4-x) /(1-x)}}{(\operatorname{Im} J)^{3}}\left(2 G^{V V}+G^{J \bar{J}}\right) W(\phi)-\left(\frac{3}{1-x}+\frac{V^{2}}{(\operatorname{Im} J)^{2}}\right) \frac{V^{(2+x) /(1-x)}}{\operatorname{Im} J} \operatorname{Re} G^{J J} W(\phi) \\
& +\frac{3}{1-x} \frac{V^{(2+x) /(1-x)} \operatorname{Re} J}{(\operatorname{Im} J)^{2}} \operatorname{Im} G^{J J} W(\phi)
\end{aligned}
$$




$$
\begin{aligned}
& -\frac{\hbar}{2} \frac{W(\phi) V^{3 /(1-x)}}{(\operatorname{Im} J)^{3}}\left[2 G^{V V}+G^{J \bar{J}}-\operatorname{Re} G^{J J}+16\left(\frac{\hbar}{2}\right)^{2}\left(V+\frac{\hbar}{2}\right)\right. \\
& \left.-\frac{12 \operatorname{Im} J V^{-1}}{1-x}\left(2 \operatorname{Im} G^{V J}-\frac{\hbar}{2} \operatorname{Im} J\right)\right] .
\end{aligned}
$$

\section{Appendix B: Fiducial volume}

For the mathematical formulation of cosmological models with non-compact spatial slices, a fiducial volume $V_{0}$ is required in order for the symplectic structure of the form $\int \mathrm{d}^{3} x p_{\phi} \dot{\phi}$ to be defined (i.e. for spatial integrals to be finite). Physical quantities must be independent of the value of $V_{0}$ and in particular remain well defined as $V_{0} \rightarrow \infty$. This has, in the past, been the cause of some confusion, so for clarity in this section we describe in detail the $V_{0}$ dependence of the all the parameters in the theory.

The canonical pair we are using is,

$$
\begin{aligned}
\{f(p) c, V\} & =\left\{f_{0} p^{x} c, \frac{3 p^{1-x}}{8 \pi G \gamma(1-x) f_{0}}\right\} \\
& =\frac{3}{8 \pi \gamma G}\{p, c\}=\frac{3}{8 \pi \gamma G} V_{0}\{\tilde{p}, \tilde{c}\}=1
\end{aligned}
$$

where the over-tilde implies that the quantity does not depend on $V_{0}$. The combination $f_{0} p^{x} c$ thus behaves as

$$
f_{0} p^{x} c=\tilde{f}_{0} \tilde{p}^{x} \tilde{c} V_{0}^{y+\frac{2 x+1}{3}}
$$

where $f_{0}=\tilde{f}_{0} V_{0}^{y}$. The freedom we have then is to fix the behavior of $f_{0}$ with respect to $V_{0}$ such that a well-defined formulation results. We could, for example, have chosen to set $y=0$, as implicitly occurred in some papers on the subject. Had we done so, we would have found:

$$
V=\frac{3 p^{1-x}}{8 \pi G \gamma(1-x) f_{0}}=\frac{3 \tilde{p}^{1-x}}{8 \pi G \gamma(1-x) \tilde{f}_{0}} V_{0}^{2(1-x) / 3}
$$

However, then we have

$$
f(p) c=f_{0} p^{x} c=\tilde{f}_{0} \tilde{p}^{x} \tilde{c} V_{0}^{(1+2 x) / 3}
$$

which only for $x=-1 / 2$ is consistent with the primary condition on the scaling behavior of $f(p)$, ensuring that holonomies are independent of $V_{0}$. In particular, if we were to attempt a limit of large $V_{0}$, holonomies or even observables such as the density at the bounce would not remain well-defined.

Instead, we must choose to have $f_{0}$ depend on $V_{0}$ as,

$$
f_{0}=\tilde{f}_{0} V_{0}^{-\frac{1+2 x}{3}}
$$

so that $f_{0} p^{x} c=\tilde{f}_{0} \tilde{p}^{x} \tilde{c}$ is independent of the regulator $V_{0}$. The parameter $f_{0}$ is $V_{0}$-independent only for $x=-1 / 2$, in which case $f_{0} p^{x} c=f_{0} \gamma \dot{a} / a$ appears in holonomies, as suggested as a special ad-hoc choice by [42]. Then we have that

$$
V=\frac{3 \tilde{p}^{1-x}}{8 \pi G \gamma(1-x) \tilde{f}_{0}} V_{0}
$$

The factor $V_{0}$ provides the correct dependence on $V_{0}$ in the Poisson bracket, Eq. (B1). In this case, taking the large- $V$ limit corresponds either to taking the large- $V_{0}$ limit or the small-discreteness limit $\left(\tilde{f}_{0} \rightarrow 0\right)$.

We are interested in physical quantities, such as the kinetic energy density at the bounce (here we focus on the $W=0$ case, the $W \neq 0$ case follows similarly), the square-root of which is given by Eq. (39),

$$
\left.\frac{H_{Q}}{\text { vol }}\right|_{\text {Bounce, largeV }}=\left[\frac{3 \tilde{p}^{-(1+2 x) / 2}}{8 \pi \gamma G(1-x)} \tilde{f}_{0} \sqrt{\frac{1+\Delta G^{V V}}{1+\Delta G^{J \bar{J}}}} \tilde{p}^{-3 / 2}\right]_{\text {Bounce, largeV }}
$$


which is independent of the fiducial volume for all choices of the lattice refinement parameters $x$ and $\tilde{f}_{0}$.

[1] M. Bojowald, Living Rev. Relativity 11, 4 (2008), http://www.livingreviews.org/lrr-2008-4.

[2] C. Rovelli, Quantum Gravity (Cambridge University Press, Cambridge, UK, 2004).

[3] T. Thiemann, Introduction to Modern Canonical Quantum General Relativity (Cambridge University Press, Cambridge, UK, 2007).

[4] A. Ashtekar and J. Lewandowski, Class. Quantum Grav. 21, R53 (2004).

[5] C. Rovelli and L. Smolin, Nucl. Phys. B 331, 80 (1990).

[6] T. Thiemann, Class. Quantum Grav. 15, 839 (1998).

[7] M. Bojowald and H. A. Kastrup, Class. Quantum Grav. 17, 3009 (2000).

[8] B. S. DeWitt, Phys. Rev. 160, 1113 (1967).

[9] D. L. Wiltshire, in Cosmology: The Physics of the Universe, edited by B. Robson, N. Visvanathan, and W. S. Woolcock (World Scientific, Singapore, 1996), pp. 473-531.

[10] M. Bojowald, Class. Quantum Grav. 18, 1071 (2001).

[11] M. Bojowald, Class. Quantum Grav. 19, 2717 (2002).

[12] M. Bojowald, Phys. Rev. Lett. 86, 5227 (2001).

[13] M. Bojowald, AIP Conf. Proc. 910, 294-333 (2007).

[14] R. Colistete Jr., J. C. Fabris, and N. Pinto-Neto, Phys. Rev. D 62, 083507 (2000).

[15] F. T. Falciano, N. Pinto-Neto, and E. S. Santini, Phys. Rev. D 76, 083521 (2007).

[16] F. Amemiya and T. Koike, Phys. Rev. D 80, 103507 (2009).

[17] M. Bojowald and A. Skirzewski, Rev. Math. Phys. 18, 713 (2006).

[18] M. Bojowald and A. Skirzewski, Int. J. Geom. Meth. Mod. Phys. 4, 25 (2007).

[19] M. Bojowald, Nature Physics 3, 523 (2007).

[20] M. Bojowald, Proc. Roy. Soc. A 464, 2135 (2008).

[21] M. Bojowald, Gen. Rel. Grav. 38, 1771 (2006).

[22] M. Bojowald, Gen. Rel. Grav. 40, 639 (2008).

[23] W. Nelson and M. Sakellariadou, Phys. Rev. D 76, 044015 (2007).

[24] W. Nelson and M. Sakellariadou, Phys. Rev. D 76, 104003 (2007).

[25] A. Barrau and J. Grain, Phys. Rev. Lett. 102, 081301 (2009).

[26] J. Grain, T. Cailleteau, A. Barrau, and A. Gorecki, Phys. Rev. D. 81, 024040 (2010).

[27] M. Shimano and T. Harada, Phys. Rev. D 80, 063538 (2009).

[28] J. F. Barbero G., Phys. Rev. D 51, 5507 (1995).

[29] G. Immirzi, Class. Quantum Grav. 14, L177 (1997).

[30] M. Bojowald, Class. Quantum Grav. 18, 1055 (2001).

[31] M. Bojowald, Class. Quantum Grav. 23, 987 (2006).

[32] M. Bojowald, Phys. Rev. D 75, 081301(R) (2007).

[33] M. Bojowald, Phys. Rev. D 75, 123512 (2007).

[34] M. Bojowald, B. Sandhöfer, A. Skirzewski, and A. Tsobanjan, Rev. Math. Phys. 21, 111 (2009).

[35] M. Bojowald and A. Tsobanjan, Phys. Rev. D 80, 125008 (2009).

[36] M. Bojowald and A. Tsobanjan, Classical Quantum Gravity 27, 145004 (2010).

[37] M. Bojowald, Gen. Rel. Grav. 40, 2659 (2008).

[38] M. Bojowald, Phys. Rev. Lett. 100, 221301 (2008).

[39] M. Bojowald and T. Strobl, Rev. Math. Phys. 15, 663 (2003).

[40] M. Bojowald, H. Hernández, and A. Skirzewski, Phys. Rev. D 76, 063511 (2007).

[41] G. Date, arXiv:1004.2952

[42] A. Ashtekar, T. Pawlowski, and P. Singh, Phys. Rev. D 73, 124038 (2006).

[43] W. Kaminski, J. Lewandowski, and T. Pawlowski, Class. Quantum Grav. 26, 035012, (2009).

[44] M. Bojowald and R. Tavakol, Phys. Rev. D 78, 023515 (2008).

[45] A. Ashtekar, T. Pawlowski, P. Singh, and K. Vandersloot, Phys. Rev. D 75, 024035 (2007).

[46] E. Bentivegna and T. Pawlowski, Phys. Rev. D 77, 124025 (2008). 